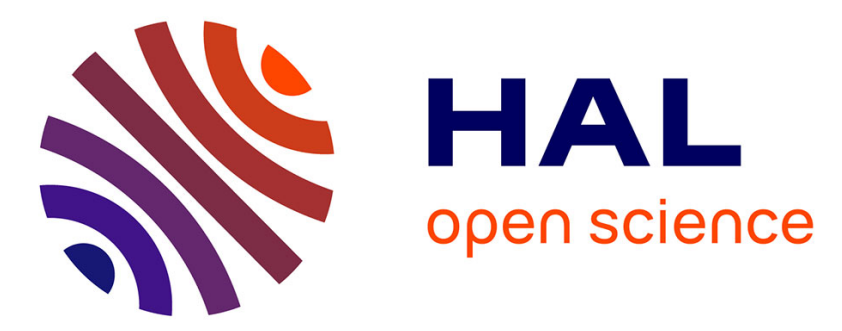

\title{
Consequences of geomagnetic activity on energization and loss of radiation belt electrons by oblique chorus waves
}

D. Mourenas, A. V. Artemyev, O. V. Agapitov, V. Krasnoselskikh

\section{To cite this version:}

D. Mourenas, A. V. Artemyev, O. V. Agapitov, V. Krasnoselskikh. Consequences of geomagnetic activity on energization and loss of radiation belt electrons by oblique chorus waves. Journal of Geophysical Research Space Physics, 2014, 119 (4), pp.2775-2796. 10.1002/2013JA019674 . insu01282024

\section{HAL Id: insu-01282024 \\ https://hal-insu.archives-ouvertes.fr/insu-01282024}

Submitted on 22 Mar 2016

HAL is a multi-disciplinary open access archive for the deposit and dissemination of scientific research documents, whether they are published or not. The documents may come from teaching and research institutions in France or abroad, or from public or private research centers.
L'archive ouverte pluridisciplinaire HAL, est destinée au dépôt et à la diffusion de documents scientifiques de niveau recherche, publiés ou non, émanant des établissements d'enseignement et de recherche français ou étrangers, des laboratoires publics ou privés.

\section{(ㅇ)(1) $\$$}

Distributed under a Creative Commons Attribution - NonCommercial - NoDerivatives 44.0 


\section{Journal of Geophysical Research: Space Physics}

\author{
RESEARCH ARTICLE \\ 10.1002/2013JA019674 \\ Key Points: \\ - Two regimes of electron energiza- \\ tion by chorus may lead to double- \\ peaked belt \\ - Variability of chorus obliq- \\ uity may explain electron \\ energization variability \\ - Oblique chorus could help parallel \\ chorus QL growth
}

Correspondence to:

D. Mourenas,

didier.mourenas@cea.fr

Citation:

Mourenas, D., A. V. Artemyev, O. V. Agapitov, and V. Krasnoselskikh

(2014), Consequences of geomagnetic activity on energization and loss of radiation belt electrons by oblique chorus waves, J. Geophys. Res. Space Physics, 119, 2775-2796, doi:10.1002/2013JA019674.

Received 29 NOV 2013

Accepted 1 APR 2014

Accepted article online 3 APR 2014

Published online 21 APR 2014

\section{Consequences of geomagnetic activity on energization and loss of radiation belt electrons by oblique chorus waves}

\author{
D. Mourenas ${ }^{1}$, A. V. Artemyev ${ }^{2,3}$, O. V. Agapitov ${ }^{2,4}$, and V. Krasnoselskikh² \\ ${ }^{1}$ CEA, DAM, DIF, F-91297, Arpajon, France, ${ }^{2}$ LPC2E/CNRS, University of Orleans, Orleans, France, ${ }^{3}$ Space Research Institute, \\ RAS, Moscow, Russia, ${ }^{4}$ National Taras Shevchenko University of Kiev, Kiev, Ukraine
}

\begin{abstract}
Statistics of amplitudes and obliquity of lower band chorus whistler mode waves have been obtained from Cluster measurements in Earth's outer radiation belt and fitted as functions of $L$, latitude, magnetic local time, and three geomagnetic activity ranges for Dst $\in[+10,-80] \mathrm{nT}$. Very oblique chorus waves have generally a much smaller average intensity than quasi-parallel waves, especially on the nightside. Nevertheless, analytical estimates and full numerical calculations of quasi-linear diffusion rates show that dayside very oblique waves $\left(\theta>60^{\circ}\right)$ dominate pitch angle scattering of energetic electrons during moderately disturbed periods. As geomagnetic activity increases, leading to higher wave amplitudes, electron lifetimes are only slightly reduced, due to a decrease of the wave obliquity probably related to Landau damping by stronger incoming fluxes from the plasma sheet. As a result, electron energization by chorus waves for Dst $>-80 \mathrm{nT}$ generally occurs in a loss-dominated regime in which energization increases at lower $L$. However, at $L \geq 6$ the most disturbed periods ( $D s t<-40 \mathrm{nT}$ ) produce a stronger energization independent of losses. Double-belt structures may therefore arise when Dst $<-40 \mathrm{nT}$, with two peaks of energization located just outside the plasmapause and at $L \sim 6$. The variability of lower band chorus wave obliquity with geomagnetic activity could actually account for some part of the observed variability of energization and loss in the outer belt. It is also suggested that quasi-linear pitch angle diffusion by very oblique waves together with energy diffusion by parallel waves might contribute to the steep wave growth observed in the day sector between the equator and $25^{\circ}$.
\end{abstract}

\section{Introduction}

Ever since the 1960s, the recognition of the threat posed by energetic particles to orbiting satellites has stimulated increasing research for better understanding the processes that control radiation belt dynamics. This is due in a large part to the risk posed by energetic electron fluxes to sattelite electronic components, as demonstrated by failures recorded during geomagnetic storms [e.g., Horne et al., 2013]. One of the most important questions regarding radiation belt physics is why some geomagnetic storms lead to an increase of the population of energetic (MeVs) electrons, while some others lead to a decrease or no change [Reeves et al., 2003; Thorne, 2010; Ni et al., 2013]. Another essential question raised early after the discovery of Earth's radiation belts is whether there is an upper limit on trapped electron fluxes and what is its actual level [Trakhtengerts, 1966; Kennel and Petschek, 1966; Summers et al., 2009, 2011]. More fundamentally, one would also like to understand the processes of whistler wave generation, whether linear [Andronov and Trakhtengerts, 1964; Kennel and Petschek, 1966] or nonlinear [Nunn et al., 1997; Omura et al., 2008].

Whistler mode chorus waves observed in the inner magnetosphere are known to be composed of many subelements of various amplitudes, some of which reaching thresholds for nonlinear effects [e.g., see Santolík et al., 2003; Cattell et al., 2008, and references therein]. Nonlinear effects such as particle trapping and phase bunching through cyclotron resonances [Tao et al., 2013, and reference therein] or Landau resonance with very oblique waves [Artemyev et al., 2012b, and reference therein] can therefore become significant for interactions with high-intensity wave packets. They certainly play an important role in the radiation belts: for instance, in relation with microburst electron precipitations [e.g., see Lakhina et al., 2010; Artemyev et al., 2012b; Tsurutani et al., 2013, and references therein]. Nevertheless, high-intensity wave packets are much more rare than lower intensity waves. After averaging over more than tens of thousands of bounce periods, the global behavior of electrons interacting with chorus waves over a wide range of latitudes and magnetic local time (MLT) can still be expected to remain at least partly akin to that due to 
quasi-linear (stochastic) diffusion by uncorrelated, moderate amplitude waves, since their average amplitude remains well below nonlinear thresholds and since bouncing particles encounter great many waves with many different amplitudes, frequencies, and wave normal angles. Moreover, the coherency of chorus wave packets has been shown to decrease sensibly with latitude [Tsurutani et al., 2011], reducing potential nonlinear effects at latitudes $\lambda>15^{\circ}-20^{\circ}$. The validity of the quasi-linear formalism for stochastic diffusion by relatively low amplitude waves has also been demonstrated by means of particle simulations [Tao et al., 2011]. While nonlinear effects undoubtedly play an important role, we shall therefore focus here on quasi-linear diffusion effects, which should determine a large part of the behavior of the trapped electrons.

The first goal of this paper is to present full statistics of Cluster spacecraft data for lower band chorus waves average intensities and wave normal angles as functions of latitude, local time, and geomagnetic activity, completing the dayside-only statistics at $L \sim 5$ provided in the work by Artemyev et al. [2013a]. This statistics has been derived from an analysis of 10 years of measurements outside the plasmasphere $(L \sim 4-8)$, and it supplements the previous statistics available in the works by Meredith et al. [2012], Agapitov et al. [2013], and Horne et al. [2013] by providing average wave intensity levels and the proportion of oblique wave intensity in three separate Dst ranges, fitted numerically by polynomials for the day and night sectors separately, and therefore directly usable in global radiation belt codes. This statistics is detailed in section 2 , where the ranges $L=4-6$ and $L=6-8$ are clearly distinguished on the basis of lower wave obliquity at higher $L$. In section 3 , analytical estimates of electron lifetimes and diffusion rates are provided for parallel and oblique chorus waves, demonstrating the increase of pitch angle diffusion by very oblique waves. The principal factor determining energization strength is then derived from a simplified model of broadening of an initially nearly cold electron distribution, showing the possible existence of two regimes of moderate and strong acceleration. On this basis, energization strength is evaluated numerically in section 4, using distributions of chorus amplitudes and wave normal angles obtained from Cluster in section 2. Two regimes of energization (loss limited and lossless) are identified and explained by a weak reduction of lifetimes as geomagnetic activity increases, due to a simultaneous decrease of the amount of very oblique waves.

The various implications of these results are then discussed in details in section 5 . The two regimes of energization at moderate and high geomagnetic activity are suggested to correspond, respectively, to low and high Landau damping of oblique waves by suprathermal electrons as well as moderate and strong parallel wave growths. We further demonstrate that these two regimes should translate spatially into two peaks of electron energization at moderate and large $L$ when $D s t<-40 \mathrm{nT}$, with the larger- $L$ maximum clearly prevailing. The variability of chorus wave obliquity (depending on many parameters) is shown to provide a possible explanation for the observed variability of electron belt energization, through its control of electron losses. Finally, it is suggested that the presence of very oblique waves during moderately active conditions could also speed up parallel wave growth (observed between the equator and medium latitudes) by increasing the anisotropy of 30-50 keV injected electrons, possibly allowing quasi-linear parallel wave growth to take over and impose new limits on such trapped electron fluxes.

\section{Spacecraft Statistics}

\subsection{Generalities}

The orbital coverage of the four Cluster spacecraft over 10 years of measurements (2001-2010) in the range $L \in[4,6]$ is fair enough to provide accurate statistics of the distributions of lower band chorus wave normal angles and root-mean-square (RMS) wave amplitudes as functions of magnetic latitude $\lambda<45^{\circ}$, magnetic local time (MLT), and geomagnetic activity in the range $-80 \mathrm{nT}<D s t<10 \mathrm{nT}$. In the domain $L \in[6,8]$, the orbital coverage was lower, yielding poorer statistics, especially concerning the most disturbed (and therefore rare) periods and near the equator. The Spatio-Temporal Analysis of Field Fluctuation-Spectrum Analyzer (STAFF-SA) experiment [Cornilleau-Wehrlin et al., 2003] on board Cluster provides Hermitian spectral matrices of the three components of the magnetic field fluctuations measured by the search coil magnetometers, calculated in the SR2 (Spin Reference) frame. Their analysis allows to derive the wave normal angle $\theta$ between the direction of wave propagation and the background geomagnetic field [Agapitov et al., 2012, 2013]. Due to the limited number of observations at a given point of space $(L, \lambda$, and MLT), the geomagnetic activity range $-80 \mathrm{nT}<D s t<10 \mathrm{nT}$ has been split into only three distinct subranges $(|D s t|<10 \mathrm{nT}$, Dst $\in[-40,-10] \mathrm{nT}$, and Dst $\in[-80,-40] \mathrm{nT})$ in order to provide meaningful statistics inside each of them. 
Only lower band chorus whistler mode waves are considered here, corresponding to a frequency $\omega$ ranging from $0.1 \Omega_{\text {ce }}$ to $0.5 \Omega_{\text {ce }}$, where $\Omega_{\text {ce }}$ denotes the electron angular gyrofrequency. Only five to seven STAFF-SA frequency channels are then available, which introduce a certain degree of uncertainty in the determination of the actual wave spectrum shape (maximum and width). Consequently, wave spectra have been assumed to be similar for parallel and oblique waves and taken from Horne et al. [2005], with a peak frequency $\omega_{m} \approx$ $0.35 \Omega_{\text {ce0 }}$ and a variance $\Delta \omega \approx 0.15 \Omega_{\text {ce0 }}$, where $\Omega_{\text {ce } 0}$ is the equatorial electron gyrofrequency (from now on, subscritpts " 0 " indicate equatorial values). Since electron lifetimes and acceleration vary weakly with the ratio $\omega_{m} / \Omega_{\text {ce0 }}$ [Mourenas et al., 2012b; Artemyev et al., 2013a], the observed variations of the latter with latitude, $L$, and MLT [e.g., see Bunch et al., 2013] are not taken into account for the sake of simplicity. A fine study of the effects of such frequency variations is left as a subject of future research.

The sensitivity of the STAFF-SA search coil magnetometers is $\sim 4 \cdot 10^{-5} \mathrm{nT} / \mathrm{Hz}^{1 / 2}$ in this frequency range [Cornilleau-Wehrlin et al., 2003]. The wave normal angle range has also been split into two parts, corresponding to very oblique $\left(\theta=60^{\circ}-90^{\circ}\right)$ and nearly parallel $\left(\theta<45^{\circ}\right)$ waves. Such a separation corresponds to the different roles played by these wave populations in particle scattering and acceleration [e.g., see Mourenas et al., 2012a, 2012b]. In particular, wave normal angles comprised between the Gendrin angle $\theta_{g} \sim \arccos \left(2 \omega / \Omega_{\mathrm{ce}}\right)$ and the resonance cone angle $\theta_{r} \sim \arccos \left(\omega / \Omega_{\mathrm{ce}}\right)$, which are most important for pitch angle scattering, lie mainly in the very oblique range such that $\theta>60^{\circ}$ at latitudes $\lambda \geq 15^{\circ}$ where they are most observed. Wave amplitudes below the sensitivity level of the STAFF-SA instruments have been excluded for evaluating the oblique to parallel wave intensity ratio. The wave normal angles have been obtained under the assumption of single plane wave propagation by the Means method [Means, 1972] (eliminating the $\sim 2 \%$ cases where the single plane wave approximation failed) and cross checked by the minimum variance analysis of magnetic field fluctuation method as described in details in the work by Agapitov et al. [2013]. This way of determining wave normal angle is equivalent to the singular value decomposition method [Santolik et al., 2003] applied to the wave magnetic field alone (a $180^{\circ}$ uncertainty on the propagation direction remains, which is unimportant here).

As concerns the cold electron density, we simply used the profile obtained by Sheeley et al. [2001] based on CRRES measurements outside of the plasmasphere, as the latter authors showed that there was little effect of geomagnetic activity on plasma density-except for an important shift of the plasmapause location. The plasmapause position was estimated based on CRRES data plotted by O'Brien and Moldwin [2003] in their Figure 2, and we used only Cluster data outside the plasmasphere. Although some nightside portion of the resulting data set could actually be on the inner edge of the plasmapause during very quiet periods (Dst $=-2$ to $-10 \mathrm{nT}$ ), the plasma density ramp is expected to be quite smooth in such cases [Carpenter and Anderson, 1992], reducing the possible error on the value of the plasma frequency $\omega_{\text {pe }}$ to gyrofrequency $\Omega_{\text {ce }}$ ratio used over this limited MLT domain. Moreover, most of the wave data would still remain outside of the plasmasphere, with higher $L$ shells corresponding to higher wave amplitudes and therefore dominating the statistics [Agapitov et al., 2013].

The distributions of lower band wave RMS amplitudes and wave normal angles $\theta$ in 2-D space $(\lambda, \theta)$ are displayed in Figure 1 for the two $L$ ranges and inside each $L$ range for the day and night sectors, as a function of geomagnetic index Dst. The domains $L \in[4,6]$ and $L=\in[6,8]$ are very different and therefore deserve to be considered separately.

\subsection{The Region $L=4-6$}

Let us first discuss the range $L \in[4,6]$. For quiet conditions $|D s t|<10 \mathrm{nT}$, a significant part of the wave power lies in the large- $\theta$ range on the dayside, although slightly less on the nightside. There are not many very oblique waves in the vicinity of the equator, but both their occurrences and average intensity rapidly increase with $\lambda$ (in agreement with ray tracing results) [see Breuillard et al., 2012; Chen et al., 2013] in both the day and night sectors. Such very oblique waves can be directly generated near the equator (as some observations suggest) [see Hayakawa et al., 1984; Lauben et al., 2002; Santolík et al., 2009; Li et al., 2013] by linear [Sauer and Sydora, 2010; Tsurutani et al., 2011] or nonlinear [Yamaguchi et al., 2013] processes, but most of them likely result from the refraction of initially quasi-parallel waves during their propagation toward higher latitudes [Thorne and Kennel, 1967; Hayakawa et al., 1984; Chen et al., 2013; Agapitov et al., 2013; Woodroffe and Streltsov, 2013]. The bottom panels of Figures $1 \mathrm{a}$ and $1 \mathrm{~b}$ show that at $\lambda \in\left[10^{\circ}, 30^{\circ}\right]$, very oblique wave amplitudes are about $10 \%-20 \%$ of parallel wave amplitudes during quiet times. At low-latitude $\lambda<15^{\circ}$, the wave normal angle distribution obtained from Cluster agrees well with the distribution provided by Time 


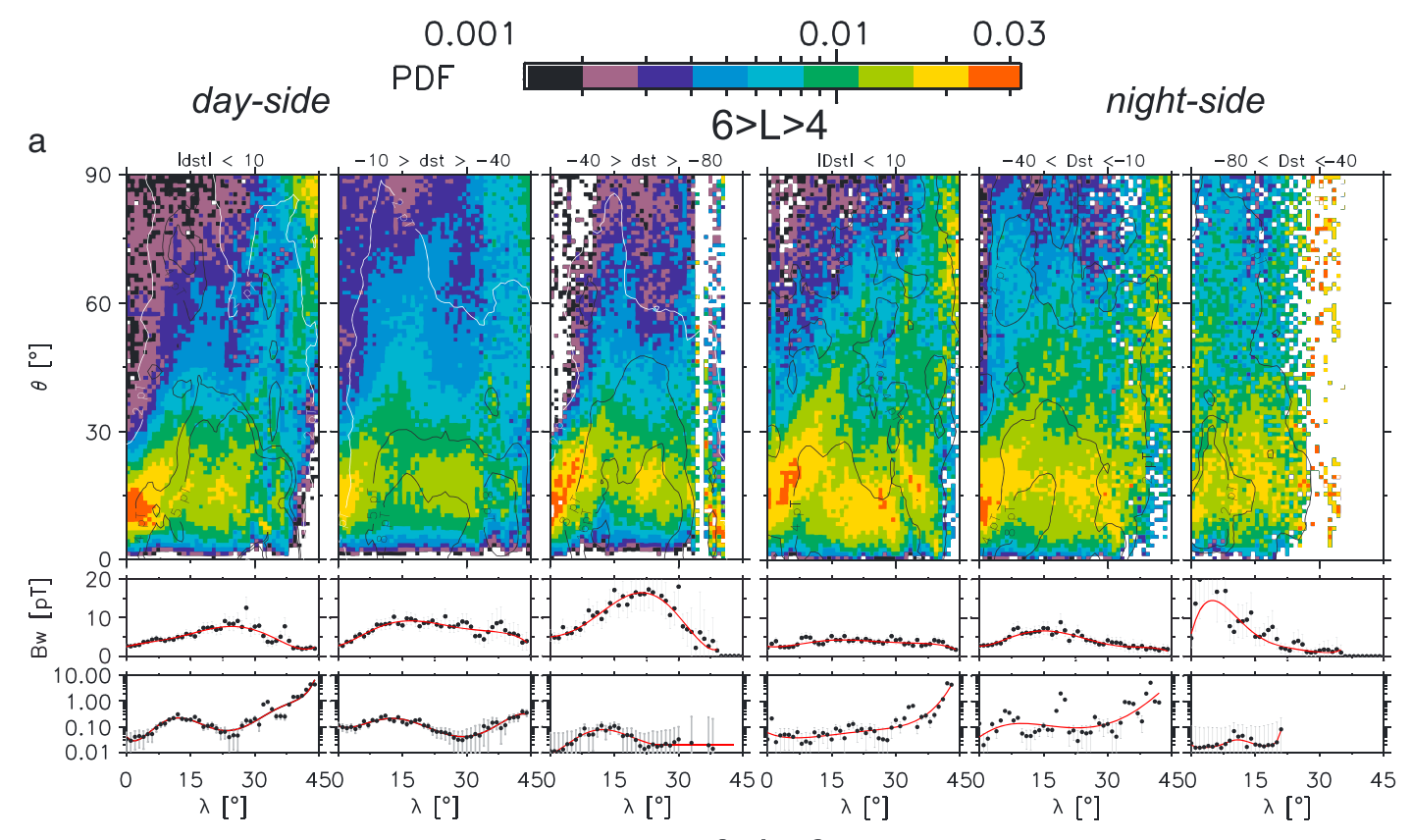

b

b $\quad 8>L>6$

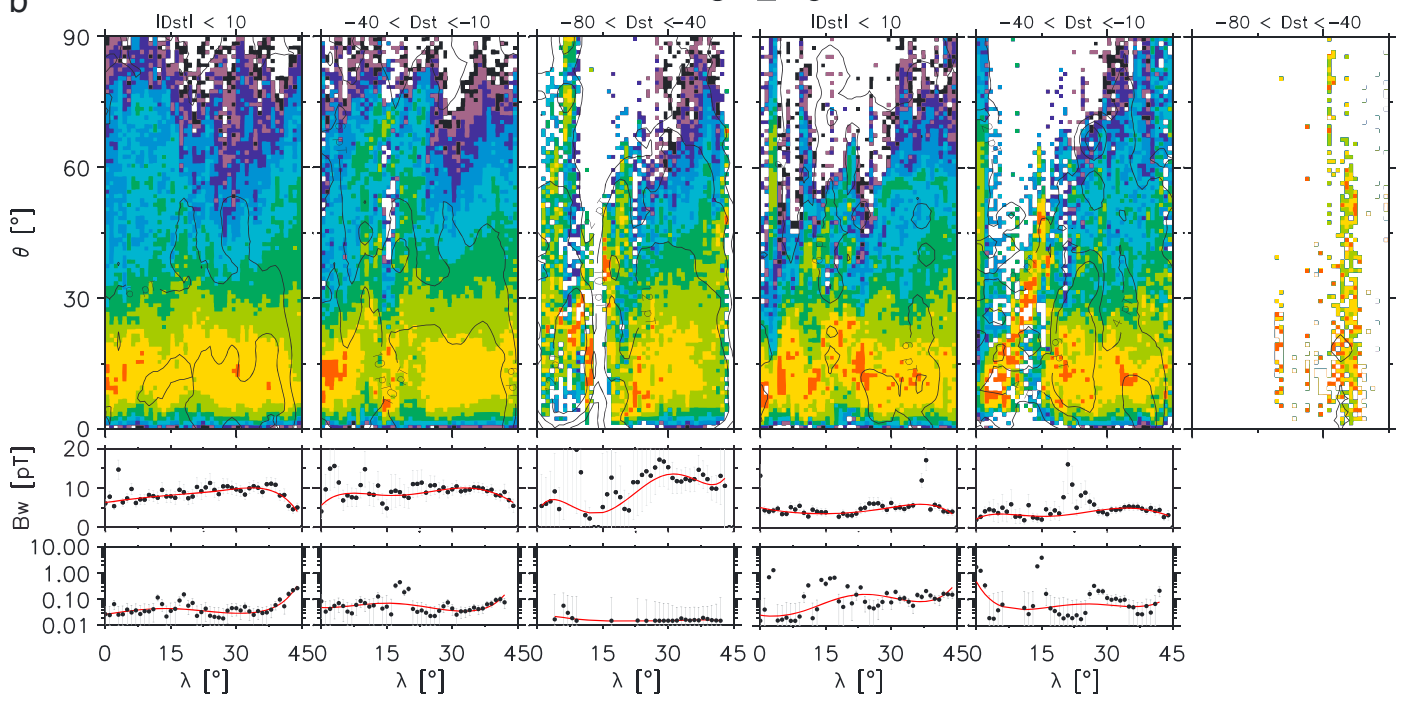

Figure 1. ( $a$ and b) Distributions of RMS wave amplitudes and wave normal angles for three Dst ranges and two $L$ ranges. Day and night sectors are presented. Upper panels in Figures $1 \mathrm{a}$ and $1 \mathrm{~b}$ show the PDF of wave occurrences as well as amplitudes in the $(\lambda, \theta)$ domain. Middle panels in Figures $1 \mathrm{a}$ and $1 \mathrm{~b}$ show the full (three components) wave amplitude as a function of latitude: spacecraft data (black circles) and approximations (red curves). Bottom panels in Figures 1a and 1b show the oblique $\left(\theta>60^{\circ}\right)$ to parallel $\left(\theta<45^{\circ}\right)$ wave amplitude ratio (using wave intensities weighted by occurrences) as a function of latitude: spacecraft data (black circles) and approximations (red curves).

History of Events and Macroscale Interactions (THEMIS) [Li et al., 2013]. In particular, the secondary peak of occurrences near $\theta=75^{\circ}$ has a very similar level relative to the main peak at low $\theta$. The THEMIS study has also shown that an important part of these very oblique waves is probably within less than $5^{\circ}$ of the resonance cone [Li et al., 2013].

At high latitudes $\left(\lambda>30^{\circ}\right)$ during quiet conditions, most of the waves are actually very oblique. Such a distribution of wave normal angles is expected to produce a significant increase of electron pitch angle scattering, because a large number of higher-order cyclotron resonances are then allowed to contribute while only the first-order one is important for quasi-parallel waves [Shklyar and Matsumoto, 2009; Artemyev et al., 2012a; Mourenas et al., 2012b]. The full wave amplitude (integrated over $\theta$ ) peaks at $\sim 5-7$ pT near $\lambda \sim 25^{\circ}$. 
At the equator or at $\lambda>40^{\circ}$, wave amplitudes are much smaller (2-3 $\mathrm{pT}$ ). The geometrical convergence of geomagnetic field lines at high latitudes cannot account for the more than threefold increase in wave intensity observed between $\lambda=0^{\circ}$ and $\lambda=15^{\circ}$ in Figure 1 in the day sector. Such a large increase of wave intensity with latitude suggests that some convective growth mechanism may be operating on the dayside: this possibility will be discussed in more details in the next sections. On the nightside, the smaller increase of the wave intensity with latitude may indicate that the energetic electron distribution is less unstable there or that Landau damping by suprathermal electrons is more efficient in this sector (which seems supported by recent studies) [see Li et al., 2010; Chen et al., 2013].

The next $|D s t|$ range $(-40<D s t(\mathrm{nT})<-10)$ corresponds to similar relative amounts of oblique waves near the equator on the dayside but smaller amounts on the nightside. Moreover, their amplitudes are now substantially smaller at high $\lambda$ ( $<30 \%$ of parallel wave amplitudes). All these observations can be explained by an increased Landau damping by incoming suprathermal electrons from the plasma sheet, an effect which has been shown to be stronger near the midnight sector during disturbed periods [e.g., Li et al., 2010]. The decrease of the proportion of oblique wave power is stronger than (or comparable to) the general increase of wave intensity with $|D s t|$, and thus, the impact of oblique waves on particle scattering slightly decreases as $|D s t|$ increases. The total wave amplitude now reaches a higher peak value of $8 \mathrm{pT}$ at $\lambda \sim 20^{\circ}$, remaining near 2-3 $\mathrm{pT}$ at the equator and at $\lambda>40^{\circ}$ as for $|D s t|<10 \mathrm{nT}$. While the RMS amplitude of oblique waves was nearly constant $\left(B_{w} \sim 2 \mathrm{pT}\right)$ at $\lambda>20^{\circ}$ for $|D s t|<10 \mathrm{nT}$ on the dayside, it now peaks at $\lambda \sim 15^{\circ}-20^{\circ}$ (being $\sim 2 \mathrm{pT}$ ), declining to $\sim 1 \mathrm{pT}$ at $\lambda>20^{\circ}$. At high latitudes, the oblique wave intensity therefore decreases 5 times as $|D s t|$ increases. However, oblique waves are still intense enough to prevail in pitch angle scattering in the day sector (see a comparison of impacts of different proportions of oblique waves in Artemyev et al. [2013b]). On the nightside, oblique wave amplitude peaks at a lower latitude $\lambda \sim 10^{\circ}$ and at a lower level ( $\sim 1.4 \mathrm{pT}$ ) than on the dayside, decreasing to $0.5-1 \mathrm{pT}$ at higher latitudes. Such a strong asymmetry between the day and night sectors (absent during quiet times) in the latitudinal distribution of oblique waves is a clear indication that Landau damping has risen due to more important injections of suprathermal electrons during the corresponding moderately disturbed periods, especially on the nightside.

Statistically, the highest $|D s t|$ range $(-80<D s t(\mathrm{nT})<-40)$ considered here corresponds to the early recovery phase of moderate to large storms, as well as the shorter (and less represented) main phase of moderate storms. In this range, oblique waves are only present at intermediate latitudes: their RMS amplitude drops to only a few percents of the total wave amplitude at $\lambda>15^{\circ}$ on both the dayside and nightside. The peak of the total wave amplitude is $\sim 15 \mathrm{pT}$ at $\lambda \sim 25^{\circ}$ on the dayside and near the equator on the nightside. The peak amplitude of lower band chorus increases roughly like $\sim \exp (|D s t| / 40)$ with $|D s t|$. The RMS amplitude of oblique waves peaks at $\sim 1 \mathrm{pT}$ at $\lambda \sim 10^{\circ}-15^{\circ}$, shrinking above $15^{\circ}$ as Landau damping has now been strongly increased even on the dayside. Since resonant electron scattering by very oblique waves is most effective at high latitudes [Artemyev et al., 2013b], the observed decrease of the oblique wave intensity at high latitudes for increasing geomagnetic activity should reduce pitch angle scattering rates at moderate equatorial pitch angles, thereby partly compensating the simultaneous increase of the total wave intensity. Since trapped electron lifetime is mainly determined by pitch angle scattering rates at small equatorial pitch angles [Albert and Shprits, 2009], it could experience relatively little decrease as geomagnetic activity rises from $D s t \sim-2 \mathrm{nT}$ to $D s t \sim-60 \mathrm{nT}$. Conversely, quasi-linear energy diffusion (acceleration) of high pitch angle particles takes place at low latitudes: it should therefore increase strongly as $B_{w}$ increases with $|D s t|$. The relevant numerical calculations will be performed in the next sections.

\subsection{The Region $L=6-8$}

Considering now the farther region $L=6$ to 8 , dayside Cluster statistics displayed in Figure 1 show that quiet time full RMS wave amplitudes reach levels similar to dayside values at $L=4.5$ to 6 for slightly more active periods such that $-40 \mathrm{nT}<D s t<-10 \mathrm{nT}$. However, the amplification of the wave intensity from the equator to $\lambda=15^{\circ}$ and $\lambda=30^{\circ}$ is more modest, about a factor of 2 and 3 , respectively. Moreover, the proportion of oblique wave power is smaller, with peaks of very oblique wave RMS amplitude at $\lambda \sim 18^{\circ}$ and $\lambda>40^{\circ}$ culminating at $1 \mathrm{pT}$ and $1.5 \mathrm{pT}$. As before in the range $L<6$, the full wave amplitude increases progressively with geomagnetic activity, while very oblique waves get more and more rapidly damped at $\lambda>10^{\circ}$.

The main differences with the range $L<6$ therefore consist in higher wave amplitudes in the vicinity the equator and a smaller population of very oblique waves. The latter is very likely due to an increased Landau damping by suprathermal (100-300 eV) electrons from the closer plasma sheet, while the former may also 
Table 1. Dayside, $L \in[4,6]^{a}$

\begin{tabular}{lccccc}
$a_{11}$ & $a_{12}$ & $a_{13}$ & $b_{11}$ & $b_{/ 2}$ & $b_{/ 3}$ \\
\hline-2.54195 & -2.34523 & -2.35857 & 2.424 & 2.684 & 4.940 \\
4.24499 & 4.27369 & 1.87555 & 3.529 & 2.799 & 1.470 \\
-3.09218 & -3.27231 & 0.245254 & -4.363 & 6.716 & 1.622 \\
0.797295 & 0.788214 & -1.18345 & 4.142 & -5.977 & 3.852 \\
-0.0631701 & -0.05399 & 0.294506 & -1.434 & 1.658 & -2.513 \\
0.0 & 0.0 & 0.0 & 0.154 & -0.154 & 0.354 \\
\hline
\end{tabular}

aCoefficients $a_{l j}$ and $b_{l j}$ for the three considered Dst ranges $(j=1,2$, and 3$)$. be the result of more unstable energetic electron populations in this region. Let us underline that for Dst < $-40 \mathrm{nT}$, the statistics from Cluster is rather poor at $L>6$, particularly near the equator and in the night sector. Considering only the most accurate range Dst > $-40 \mathrm{nT}$, however, one finds that average intensities are on average about 3 times larger for $L \sim 7$ than for $L \sim 5$ to 6 , in general agreement with previous THEMIS statistics [Li et al., 2009]. On the nightside, full wave amplitudes and oblique to parallel amplitude ratios are similar to nightside values at $L \sim 5-6$ (cf. Figure 1).

For $L \in[6,8]$, Cluster's sparse data in the highest $|D s t|$ range $(-80 \mathrm{nT}<D s t<-40 \mathrm{nT})$ on the nightside only allows to show that the oblique wave content is almost null, as in the region $L<6$ for the same Dst range. This leaves us with no other choice but to supplement Cluster data in this domain by some other satellite data. Fortunately, a recent study by Spasojevic and Shprits [2013] provides numerical fits to lower band chorus RMS amplitudes $B_{w}$ obtained from electric fluctuation $E_{w}$ measurements on board CRRES, under the assumption of parallel wave propagation. Since we know from Cluster that it is indeed the case at $L \sim 7$ during high- $|D s t|$ periods, we have decided to make use of these fits from CRRES to complement Cluster ones. However, comparing CRRES and Cluster statistics of RMS wave amplitudes $B_{w}$ in the day sector at $L \sim 7$ (where both data were available) and for $K_{p} \sim 5$ (roughly corresponding to the same $D s t$ range) [see Yermolaev and Yermolaev, 2003] has revealed a factor of $\sim 2$ discrepancy. Incidentally, Meredith et al. [2012] recently compared Cluster, THEMIS, and CRRES wave amplitudes $B_{w}$ (similarly deduced from $E_{w}$ ) near $L=6$ and found Cluster and THEMIS measurements to agree well, while CRRES amplitudes on the nightside appeared generally larger than THEMIS ones by factors $\sim 2$. For the sake of consistency with the data from Cluster used throughout the present study, CRRES RMS amplitudes $B_{w}$ have therefore been divided by 2 in order to roughly coincide with Cluster measurements of magnetic field fluctuations. Some possible explanations for this discrepancy stem from (1) the fact that the CRRES database covers only 1 year in 1990-1991 with more nightside coverage [Spasojevic and Shprits, 2013; Horne et al., 2013], while the Cluster database covers almost a full solar cycle in 2001-2011 with more dayside coverage, and (2) the likely overestimation of the actual value of $B_{w}$ in CRRES fits [Spasojevic and Shprits, 2013] as their assumption $B_{w} / E_{w}=N_{\theta=0}$ (with $N$ the cold plasma refractive index) becomes erroneous for $\theta>20^{\circ}$ [see Lengyel-Frey et al., 1994].

In the remainder of this paper, we shall use the data presented above to calculate pitch angle and energy diffusion coefficients. To this aim, the $\theta$ distribution has been approximated by two Gaussians with mean values and variances depending on $\lambda: g(\theta, \lambda)=g_{1}(\theta, \lambda)+10^{2 A(\lambda)} g_{2}(\theta, \lambda)$ where $g_{i}=\exp \left(-\left(\theta-\theta_{\mathrm{mi}}(\lambda)\right)^{2} /\right.$ $\left.\theta_{\mathrm{wi}}^{2}(\lambda)\right)$ and $i=1,2$. $A(\lambda)$ is derived from mean-square-root fits to the ratios shown in the bottom panels of Figures $1 \mathrm{a}$ and $1 \mathrm{~b}: A^{j}=\sum_{l=0}^{l=4} a_{l j}\left(\lambda / 10^{\circ}\right)^{l}$ (coefficients $a_{l j}$ are given in Tables $1-4$ ) where $j=1,2$, and 3 corresponds to three $D$ st ranges. All these approximations can be used only within defined ranges of latitudes; see Table 5 . Since functions $\theta_{\mathrm{mi}}(\lambda)$ and $\theta_{\text {wi }}(\lambda)$ were found to vary only weakly with $\lambda$ and Dst, we simply took them as constants: $\theta_{m 1}(\lambda) \approx 15^{\circ}, \theta_{m 2}(\lambda) \approx 75^{\circ}$, and $\theta_{w 1}(\lambda) \approx \theta_{w 2}(\lambda) \approx 10^{\circ}$. The full RMS wave amplitude $B_{w}$ is also provided in the same Tables $1-4$ under the form of polynomial fits $B_{w}^{j}=\sum_{l=0}^{l=5} b_{l j}\left(\lambda / 10^{\circ}\right)^{l}$ to the data displayed in Figure 1.

Table 2. Nightside, $L \in[4,6]^{\text {a }}$

\begin{tabular}{lccccc}
$a_{l 1}$ & $a_{12}$ & $a_{13}$ & $b_{l 1}$ & $b_{12}$ & $b_{l 3}$ \\
\hline-1.18010 & -2.30635 & -2.28895 & 2.587 & 2.627 & 5.856 \\
0.895675 & 3.65788 & -6.68158 & -1.573 & -0.013 & 41.86 \\
-0.83162 & -2.89702 & 17.4566 & 5.328 & 8.170 & -65.41 \\
0.211308 & 0.777997 & -13.5627 & -3.581 & -6.503 & 36.88 \\
-0.00936 & -0.06302 & 3.27594 & 0.924 & 1.726 & -9.24 \\
0.0 & 0.0 & 0.0 & -0.084 & -0.153 & 0.867 \\
\hline \multicolumn{2}{c}{ aCoefficients $a_{l j}$ and $b_{l j}$ for the three considered Dst ranges } \\
$(j=1,2$, and 3).
\end{tabular}

\section{Analytical Estimates of Electron Lifetimes and Related Energization Regimes}

Starting from the usual formulation of electron quasi-linear diffusion rates [Lyons et al., 1971, 1972; Lyons, 1974; Glauert and Horne, 2005; Albert, 2005], 
Table 3. Dayside, $L \in[6,8]^{\text {a }}$

\begin{tabular}{lccccc}
$a_{11}$ & $a_{12}$ & $a_{13}$ & $b_{11}$ & $b_{/ 2}$ & $b_{13}$ \\
\hline-1.81859 & -1.47559 & -1.8239 & 6.330 & 5.601 & 1.565 \\
0.123943 & -0.08905 & 0.0 & 0.870 & 10.99 & 52.94 \\
0.408486 & 0.359753 & 0.0 & 1.654 & -13.50 & -62.81 \\
-0.271584 & -0.306 & 0.0 & -1.713 & 6.742 & 31.45 \\
0.04281 & 0.0534596 & 0.0 & 0.634 & -1.401 & -6.982 \\
0.0 & 0.0 & 0.0 & -0.079 & 0.0998 & 0.567 \\
\hline
\end{tabular}

aCoefficients $a_{l j}$ and $b_{l j}$ for the three considered Dst ranges $(j=1,2$, and 3$)$.

an approximate analytical expression of the bounce-averaged pitch angle scattering rate $\left\langle D_{\alpha \alpha}\right\rangle_{B}$ in the equatorial pitch angle range $\alpha_{0}<35^{\circ}$ can be derived under the assumption of quasi-parallel $\left(\theta<45^{\circ}\right)$ lower band chorus waves [Mourenas et al., 2012b; Artemyev et al., 2013b], yielding

$$
\left\langle D_{\alpha \alpha}\right\rangle_{B, \theta<45^{\circ}} \approx \frac{B_{w}^{2} \Omega_{\mathrm{ce} 0}^{10 / 3} /\left(\omega_{m}^{7 / 9} \Omega_{\mathrm{pe}}^{14 / 9}\right)}{15 B_{0}^{2} \gamma p^{14 / 9} \cos ^{2} \alpha_{0}},
$$

where $\omega_{m}$ is the mean chorus frequency and $B_{0}$ the equatorial value of the geomagnetic field. The corresponding parallel waves lifetime reads as [Mourenas et al., 2012b]

$$
\tau_{L, \theta<45^{\circ}}[\mathrm{s}] \approx \frac{220\left[\mathrm{pT}^{2} \cdot \mathrm{s}^{2} / \mathrm{rad}\right]}{B_{w}^{2}} \frac{p^{14 / 9} \gamma \omega_{m}^{7 / 9} \Omega_{\mathrm{pe}}^{14 / 9}}{\Omega_{\mathrm{ce} 0}^{12 / 9}},
$$

where from now on bounce-averaged RMS wave amplitude $B_{w}$ is in picotesla, angular frequencies are in $\mathrm{rad} / \mathrm{s}, \gamma$ is the relativistic factor, and $p=\left(\gamma^{2}-1\right)^{1 / 2}$. Now, assume that quasi-parallel waves are mixed with a significant amount of very oblique waves $\left(\theta>60^{\circ}\right)$. Very oblique wave amplitudes need not be comparable to parallel wave amplitudes. They merely need to satisfy the condition

$$
\begin{aligned}
& \int_{\tan \theta_{g}}^{\tan \theta_{r}} B_{w, \theta>\theta_{g}}^{2} \tan \theta \sqrt{1+\tan ^{2} \theta} \mathrm{d} \tan \theta \\
& \geq \int_{0}^{1} B_{w, \theta<45^{\circ}}^{2} \tan \theta \sqrt{1+\tan ^{2} \theta} \mathrm{d} \tan \theta
\end{aligned}
$$

for their actual, $\theta$-integrated wave power [see Lyons et al., 1971] to dominate in diffusion coefficients [Mourenas et al., 2012b; Artemyev et al., 2013b]. Thus, very oblique waves may well be measured with much smaller average amplitudes than parallel waves and yet prevail in scattering rates because of the steep increase of $\tan \theta$ for $\theta>60^{\circ}$, especially at some distance from the equator where $\tan \theta_{r} \sim \Omega_{\text {ce }}(\lambda) / \omega_{m}$ increases fast with latitude. Cluster observations show precisely that very oblique waves are often observed with significant amplitudes for $\lambda \in\left[15^{\circ}, 40^{\circ}\right]$ on the dayside during moderatetly disturbed periods.

When such a significant amount of very oblique waves is present only at $\lambda<20^{\circ}$, analytical estimates as well as numerical calculations show that lifetimes are roughly equal to half the parallel wave lifetime [Artemyev et al., 2013a, 2013b]. However, if very oblique chorus waves are present up to high latitudes, the diffusion rates are profoundly modified and take a form [Mourenas et al., 2012b; Artemyev et al., 2013a]

$$
\left\langle D_{\alpha \alpha}\right\rangle_{B, \theta>60^{\circ}} \approx \frac{2 B_{w}^{2} \Omega_{\mathrm{ce} 0}^{2}}{3 B_{0}^{2} \gamma p \Omega_{\mathrm{pe}} \sin \alpha_{0}}
$$

Table 4. Nightside, $L \in[6,8]^{\text {a }}$

\begin{tabular}{lccc}
$a_{l 1}$ & $a_{l 2}$ & $b_{l 1}$ & $b_{/ 2}$ \\
\hline-1.99353 & -0.329464 & 5.058 & 2.156 \\
-0.773695 & -3.01540 & -2.694 & 5.639 \\
1.71804 & 2.43066 & 2.022 & -8.223 \\
-0.697231 & -0.7444 & -1.056 & 4.470 \\
0.082615 & 0.07739 & 0.367 & -0.967 \\
0.0 & 0.0 & -0.046 & 0.071 \\
\hline
\end{tabular}

${ }^{\mathrm{a} C o e f f i c i e n t s} a_{l j}$ and $b_{l j}$ for the three considered Dst ranges $(j=1,2$, and 3$)$. in the range $\alpha_{0}>10^{\circ}-20^{\circ}$. The corresponding analytical estimate of $\tau_{L}$ is [Mourenas et al., 2012b; Artemyev et al., 2013a]

$$
\tau_{L, \theta>60^{\circ}}[\mathrm{s}] \approx \frac{35\left[\mathrm{pT}^{2} \cdot \mathrm{s}^{2} / \mathrm{rad}\right] \gamma p \Omega_{\mathrm{pe}}}{B_{w}^{2}} .
$$

Only the first-order cyclotron resonance contributes to scattering at small $\alpha_{0}$ for quasi-parallel waves. The corresponding $\left\langle D_{\alpha \alpha}\right\rangle_{B, \alpha_{0}<45^{\circ}}$ decreases toward the loss cone like $1 / \cos ^{2} \alpha_{0}$. On the contrary, 
Table 5. Latitude Ranges Where Coefficients From Tables 1-4 Can be Used $^{\text {a }}$

\begin{tabular}{lcccc} 
& $\begin{array}{c}\text { Dayside } \\
L \in[4,6]\end{array}$ & $\begin{array}{c}\text { Nightside } \\
L \in[4,6]\end{array}$ & $\begin{array}{c}\text { Dayside } \\
L \in[6,8]\end{array}$ & $\begin{array}{c}\text { Nightside } \\
L \in[6,8]\end{array}$ \\
\hline Parallel waves & & & & \\
$\mid$ Dst $\mid<10$ & $45^{\circ}$ & $45^{\circ}$ & $45^{\circ}$ & $40^{\circ}$ \\
$-40<D s t<-10$ & $45^{\circ}$ & $45^{\circ}$ & $45^{\circ}$ & $40^{\circ}$ \\
$-80<D s t \mid-40$ & $40^{\circ}$ & $35^{\circ}$ & $40^{\circ}$ & \\
Oblique waves & & & & \\
$\mid$ Dst $\mid<10$ & $45^{\circ}$ & $43^{\circ}$ & $45^{\circ}$ & $40^{\circ}$ \\
$-40<$ Dst $<-10$ & $45^{\circ}$ & $43^{\circ}$ & $43^{\circ}$ & $40^{\circ}$ \\
$-80<$ Dst <-40 & $30^{\circ}$ & $20^{\circ}$ & $40^{\circ}$ & \\
\hline
\end{tabular}

${ }^{\mathrm{a}}$ For oblique waves within the range $-80 \mathrm{nT}<D s t<-40 \mathrm{nT}$, dayside, $L \in[4,6]$ the coefficient $A$ is equal to -1.3114 for $\lambda \in\left[30^{\circ}, 40^{\circ}\right]$. scattering by very oblique waves increases like $1 / \sin \alpha_{0}$, leading to a large reduction of electron lifetimes by a factor $\sim 4 N_{r}^{2 / 3}$ due to the larger number $\sim 4 N_{r} \sim$ $8 p \Omega_{p e 0} \omega_{m} / \Omega_{\text {ce0 }}^{2}$ of contributing higher-order positive and negative cyclotron resonances [Mourenas et al., 2012b; Artemyev et al., 2013b].

The effect of oblique waves on energy diffusion rates is weaker, due to the $1 / n^{2}$ decrease of the contribution of higher-order cyclotron resonances with resonance number $n$ [Lyons, 1974; Mourenas et al., 2012a]. Assum-

ing that this effect remains small indeed, the quasi-linear energy diffusion rate of electrons by parallel chorus waves at $\alpha_{0}>45^{\circ}$ can be written as

$$
\begin{aligned}
\left\langle\frac{D_{\mathrm{EE}}}{E^{2}}\right\rangle_{B, \alpha_{0}>45^{\circ}}\left[\mathrm{s}^{-1}\right] \approx & \frac{B_{w}^{2} \Omega_{\mathrm{ce} 0}^{3 / 2} \omega_{m}^{1 / 2}}{100\left[\mathrm{pT}^{2} \cdot \mathrm{s}^{2} / \mathrm{rad}\right]} \\
& \times \frac{(\gamma+1)^{1 / 2} \sin \alpha_{0}}{\tan \Delta \theta \Omega_{\mathrm{pe}}^{3} \gamma(\gamma-1)^{3 / 2}},
\end{aligned}
$$

where $\Delta \theta \sim 30^{\circ}$ is a realistic estimate of lower band chorus wave normal angle spread [Mourenas et al., 2012a] and $B_{w}^{2}$ is the average wave intensity at latitudes $\lambda<\sqrt{2} /\left(3 \tan \alpha_{0}\right)$ before the mirror points (i.e., at $\lambda=0^{\circ}-15^{\circ}$ for $\alpha_{0} \sim 55^{\circ}-80^{\circ}$ ). For $\alpha_{0}<45^{\circ}$ and quasi-parallel waves, only the first-order cyclotron resonance contributes and $\left\langle D_{\mathrm{EE}}\right\rangle_{B}$ can be obtained by simply multiplying the full expression for $\left\langle D_{\alpha \alpha}\right\rangle_{B}[$ Mourenas et al., 2012b] by $(\gamma+1)^{2} \sin ^{2} \alpha_{0} \cos ^{2} \alpha_{0} \omega_{m}^{2} / \Omega_{\text {ce0 }}^{2}$ [Mourenas et al., 2012a]. For not-too-high electron energy such that $E(\mathrm{MeV})<10 \Omega_{\mathrm{ce} 0}^{3} /\left(\Omega_{p e 0}^{2} \omega_{m}\right)$, a simplified expression can be derived, which reads as

$$
\begin{aligned}
\left\langle\frac{D_{\mathrm{EE}}}{E^{2}}\right\rangle_{B, \alpha_{0}<45^{\circ}}\left[\mathrm{s}^{-1}\right] \approx & \frac{B_{w}^{2} \omega_{m}^{23 / 18}}{260\left[\mathrm{pT}^{2} \cdot \mathrm{s}^{2} / \mathrm{rad}\right]} \\
& \times \frac{(\gamma+1)^{23 / 18} \sin ^{2} \alpha_{0}}{\Omega_{\mathrm{ce} 0}^{5 / 6} \Omega_{p e 0}^{13 / 9} \gamma(\gamma-1)^{13 / 18}}
\end{aligned}
$$

For $\alpha_{0} \sim 15^{\circ}-45^{\circ}$, a rough estimate can be obtained by substituting in the preceding expression $\alpha_{0}=30^{\circ}$ and the average value of $B_{w}^{2}$ at latitudes $\lambda \sim 5^{\circ}-30^{\circ}$. Note that all the above simplified analytical expressions are valid only when cyclotron resonance with parallel waves exists, i.e., for $E(\mathrm{MeV})>\Omega_{\text {ce } 0}^{3} /\left(4 \Omega_{p e 0}^{2} \omega_{m}\right)$. For $L=4-7$, it corresponds to $E \geq 20-30 \mathrm{keV}$, while their upper limit of validity is roughly $E<2 \mathrm{MeV}$.

Both $\left\langle D_{\mathrm{EE}}\right\rangle_{B}$ and $\tau_{L}$ vary with electron energy like $\sqrt{E}$ for $E \leq 0.5 \mathrm{MeV}$ and $\alpha_{0}>45^{\circ}$. Let us assume to first order that they are both weakly varying with $E$. As suggested by Horne et al. [2005], the evolution of the trapped electron distribution function $F$ can then be described approximately by a Fokker-Planck equation with quasi-linear bounce-averaged isotropic energy and pitch angle diffusion coefficients (provided that mixed diffusion can be neglected, which should be appropriate only for wide $\theta$ distributions) [see Albert, 2009]. Now, many recent observations in the outer radiation belt have shown that pronounced high-energy electron dropouts frequently occur during the initial, main phase of storms [Turner et al., 2013; Ni et al., 2013]. This allows us to consider simplified analytical solutions proposed by Balikhin et al. [2012] for an initially cold distribution without high-energy electrons. Taking into account finite lifetimes, the early time broadening of the electron distribution takes a simple form [Artemyev et al., 2013a]

$$
F(t) \propto E^{-4(1-\eta)} t^{-\eta} \exp \left(-\frac{E^{2}}{4\left\langle D_{\mathrm{EE}}\right\rangle_{B} t}-\frac{t}{\tau_{L}}\right),
$$


with $\eta \sim 5 / 4$ for $E<0.5 \mathrm{MeV}$ and $\eta \sim 3 / 2$ for $E>1 \mathrm{MeV}$ [see also Bakhareva, 2005]. A careful inspection of (7) shows that at high energy the electron distribution $F$ reaches a maximum at a time $t_{\max }$ given by

$$
t_{\max } \approx \frac{\tau_{L}}{2}\left(-\eta+\sqrt{\eta^{2}+\frac{E^{2}}{\left\langle D_{\mathrm{EE}}\right\rangle_{B} \tau_{L}}}\right) .
$$

An important factor to estimate electron energization is therefore the term $\tau_{L}\left\langle D_{\mathrm{EE}}\right\rangle_{B} / E^{2}$. The range $\tau_{L}\left\langle D_{\mathrm{EE}}\right\rangle_{B} / E^{2}>1$ corresponds to a regime of negligible losses where the maximum of $F$ is reached at $t_{\max } \sim E^{2} /\left(4 \eta\left\langle D_{\mathrm{EE}}\right\rangle_{B}\right)$ [Balikhin et al., 2012]. On the other hand, the domain $\tau_{L}\left\langle D_{\mathrm{EE}}\right\rangle_{B} / E^{2} \ll 1$ corresponds to a regime of loss-dominated electron energization. Then, one gets $t_{\max } \sim \tau_{L} /\left(2 \sqrt{\tau_{L}\left\langle D_{\mathrm{EE}}\right\rangle_{B} / E^{2}}\right)$ and energization increases with $\tau_{L}\left\langle D_{\mathrm{EE}}\right\rangle_{B} / E^{2}$, since the maximum value of $F$ varies like $\exp \left(-1 / \sqrt{\tau_{L}\left\langle D_{\mathrm{EE}}\right\rangle_{B} / E^{2}}\right)[$ Artemyev et al., 2013a]. Actually, the high-energy electron flux nearly reaches its maximum already at $t \sim t_{\max } / 3$ and remains roughly constant as long as injections of lower energy electrons from the plasma sheet continue, before decreasing [e.g., see Bakhareva, 2005]. The loss-dominated regime scaling law $t_{\max } \propto E \gamma$ is actually close to the approximate scaling $\Delta t \propto E$ of the time delay between electron energization and solar wind velocity changes obtained by Balikhin et al. [2012] for $0.1 \mathrm{MeV} \leq E \leq 1 \mathrm{MeV}$ from Los Alamos National Laboratory geosynchronous satellite measurements.

\section{Numerical Results: Two Regimes of Electron Energization Depending on Dst and $L$}

It is worth noting that the experimental wave normal angle distribution $g(\theta)$ measured by Cluster is actually equivalent to $g(\theta) \sin \theta$ in the usual formulation of the diffusion rates [Lyons et al., 1971; Glauert and Horne, 2005; Albert, 2005]. Consequently, the integrals over $\theta$ must be performed after suppressing in their expression one $\sin \theta$ term which is already included in our $g(\theta)$ distribution (as already done in the works by Artemyev et al. [2013a] and Li et al. [2013]). Wave normal angle distributions $g(\theta, \lambda)$ consisting of two Gaussians are used to calculate diffusion rates, together with the Appleton-Hartree cold plasma whistler mode dispersion relation [Artemyev et al., 2013b]. The numerical scheme has been explicited in the works by Horne et al. [2005] and Mourenas et al. [2012b]. We consider either the simplified case of a constant plasma frequency along geomagnetic field lines or a more realistic increase $\Omega_{\mathrm{pe}} \sim \cos ^{-2} \lambda$. The latter scaling has been shown by Denton et al. [2006] to be a good power law fit to the experimentally determined density variation for $L \in[4,8]$ and $\lambda<45^{\circ}$. Although thermal effects, which essentially affect here very oblique waves [Hashimoto et al., 1977], are not formally included in our calculations, two important upper bounds on the wave refractive index $N$ have been imposed $\left(N<300\right.$ and $\left.\tan \theta<0.995 \tan \theta_{r}\right)$. It is shown in Appendix A that using such bounds should yield reliable diffusion rates for cold plasma effective temperatures $T_{\text {eff }} \sim 1 \mathrm{eV}$ to $10 \mathrm{eV}$ at $L \in[4,6]$, i.e., for low but realistic plasma $\beta$ values $\beta \sim 4 \cdot 10^{-5}$ to $4 \cdot 10^{-4}$. Moreover, thermal effects on diffusion rates turn out to be smaller for higher-energy electrons $(E \sim 1 \mathrm{MeV})$ than for lower energy ones $(E \sim 0.1 \mathrm{MeV})$, as the main contributions to pitch angle scattering come from lower $N$ values and thus from waves farther away from the resonance cone angle, less likely to be damped (see Appendix A for details). At $L>6$, thermal effects should be similar or smaller due to lower occurrences of very oblique waves.

The numerical bounce-averaged diffusion rates $\left\langle D_{\alpha \alpha}\right\rangle_{B} / p^{2}$ and $\left\langle D_{\mathrm{EE}}\right\rangle_{B} / E^{2}$ are plotted in Figure 2 for $E=100 \mathrm{keV}$ in the three considered $D s t$ ranges at $L \sim 4-6$ in the day and night sectors. One can see that $\left\langle D_{\alpha \alpha}\right\rangle_{B}$ retains essentially the same slope in $1 / \sin \alpha_{0}$ in the range $10 \mathrm{nT}>$ Dst $>-40 \mathrm{nT}$ in both the day and night sectors, which is representative of a prevalence of very oblique wave scattering as in equation (3) [Mourenas et al., 2012b]. Nonetheless, the small- $\alpha_{0}$ part of $\left\langle D_{\alpha \alpha}\right\rangle_{B}$ is $4-5$ times higher on the dayside due to higher intensities of very oblique waves there at $\lambda>10^{\circ}$. In the most perturbed case $-40 \mathrm{nT}<D$ st $<-80 \mathrm{nT}$, conversely, $\left\langle D_{\alpha \alpha}\right\rangle_{B}$ near $\alpha_{0}=75^{\circ}$ is larger by factor 4 on the dayside and by factor 30 on the nightside due to higher quasi-parallel wave amplitudes at low latitudes. Simultaneously, stronger Landau damping of oblique waves by more intense fluxes of incoming suprathermal electrons during geomagnetically active periods [Li et al., 2010; Chen et al., 2013] leads to smaller levels of $\left\langle D_{\alpha \alpha}\right\rangle_{B}$ near the loss cone edge. As a result, the profile of $\left\langle D_{\alpha \alpha}\right\rangle_{B}$ is strongly modified. On the dayside it becomes roughly a plateau, indicating that some very oblique waves are still present. On the nightside, the slope is actually reversed, now varying roughly like $1 / \cos ^{2} \alpha_{0}$ as it must be the case for scattering by quasi-parallel waves only (see equation (1)). Indeed, very oblique waves are almost absent in this case (see Figure 1). The energy diffusion rate $\left\langle D_{\mathrm{EE}}\right\rangle_{B} / E^{2}$, which 

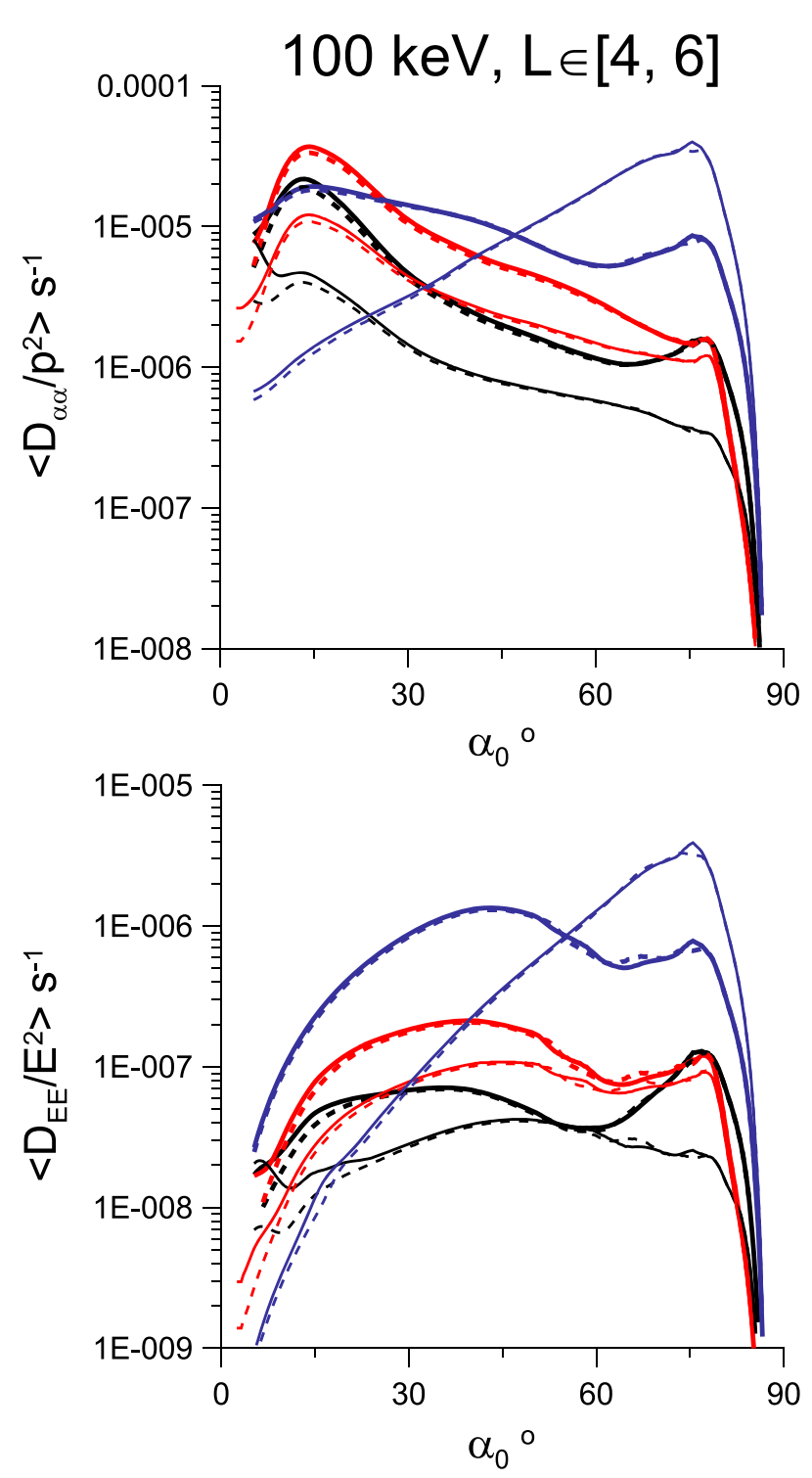

Figure 2. Bounce-averaged pitch angle and energy diffusion coefficients as a function of $\alpha_{0}$ for three Dst ranges and $E=100 \mathrm{keV}$ in the day (wide lines) and night (thin lines) sectors for $L=4$ to 6 . Black, red, and blue curves correspond, respectively, to $|D s t|<10 \mathrm{nT}$, Dst $\in[-40,-10] \mathrm{nT}$, and Dst $\in$ $[-80,-40] \mathrm{nT}$. The cases of constant (solid curves) and realistic latitudinally varying density (dashed curves) are considered. is mainly determined by quasi-parallel waves (see section 3 ) constitutes a plateau in the night sector for Dst $>-40 \mathrm{nT}$ and in the day sector for $|D s t|<10 \mathrm{nT}$. In the day sector for $D s t<-10 \mathrm{nT}$, $\left\langle D_{\mathrm{EE}}\right\rangle_{B} / E^{2}$ has a broad maximum for $\alpha_{0}=30^{\circ}-50^{\circ}$ related to the latitudinal peak of quasi-parallel wave intensity near $\lambda \sim 25^{\circ}$ seen in Figure 1. An increase of $\left\langle D_{\mathrm{EE}}\right\rangle_{B} / E^{2}$ by a factor of 5 is observed between the medium and highly disturbed periods. During the most perturbed periods considered here (Dst $\in$ $[-80,-40] \mathrm{nT})$ on the nightside, $\left\langle D_{\mathrm{EE}}\right\rangle_{B} / E^{2}$ again increases by a factor of 30 at high $\alpha_{0}$, which is again due to higher wave amplitudes at low latitudes.

The bounce-averaged and MLT-averaged $\left\langle D_{\alpha \alpha}\right\rangle_{B}$, lifetime $\tau_{L}$, and $\tau_{L}\left\langle D_{\mathrm{EE}}\right\rangle_{B} / E^{2}$ are all plotted in Figure 3 for $E=0.1$ and $1 \mathrm{MeV}$ in the three considered Dst ranges at $L \sim 4-6$. Lifetimes are derived by numerical $\alpha_{0}$ integration of $1 /\left(4\left\langle D_{\alpha \alpha}\right\rangle_{B} \tan \alpha\right)$ from the loss cone angle up to $\sim 83^{\circ}$ [Albert and Shprits, 2009; Mourenas et al., 2012b], considering that other kinds of observed waves, such as upper band chorus, fast magnetosonic, or lower frequency whistlers, although less intense, should partly fill the gap in pitch angle diffusion near $90^{\circ}$ for $E \sim 100 \mathrm{keV}$ electrons [e.g., see Shprits, 2009; Meredith et al., 2012;

Artemyev et al., 2013b]. In both pitch angle and energy diffusion rates, dayside scattering clearly dominates in the range Dst $\in[-40,10] \mathrm{nT}$ of moderate geomagnetic activity, showing increased diffusion at smaller pitch angles. But nightside scattering clearly prevails at $\alpha_{0}>45^{\circ}$ during important disturbances (Dst $\in[-80,-40] \mathrm{nT}$ ), while dayside scattering remains dominant at $\alpha_{0}<45^{\circ}$. This makes for broad maxima from $\alpha_{0} \sim 15^{\circ}$ to $80^{\circ}$ in both $\left\langle D_{\alpha \alpha}\right\rangle_{B}$ and $\left\langle D_{\mathrm{EE}}\right\rangle_{B} / E^{2}$ in all circumstances. Nevertheless, particle scattering is more important at $\alpha_{0}<45^{\circ}$ when Dst $\in[-40,10] \mathrm{nT}$ (due to the input of very oblique waves), while particle scattering at $\alpha_{0}>45^{\circ}$ becomes dominant when Dst $\in[-80,-40] \mathrm{nT}$ (due to the suppression of oblique waves and the concomitant increase of low-latitude quasi-parallel wave intensity).

It is worth noting that all the cases studied here on the basis of lower band chorus RMS amplitudes obtained from 10 years of Cluster statistics [Agapitov et al., 2013] for $L \in[4,6]$ correspond to the regime $\tau_{L}\left\langle D_{\mathrm{EE}}\right\rangle_{B} / E^{2} \ll 1$ of loss-limited electron energization. This contrasts with some previous works where energization rates exceeded loss rates as a consequence of an assumption of parallel chorus in lifetime calculations, as well as due to higher low-latitude to medium-latitude wave intensity ratios in the 

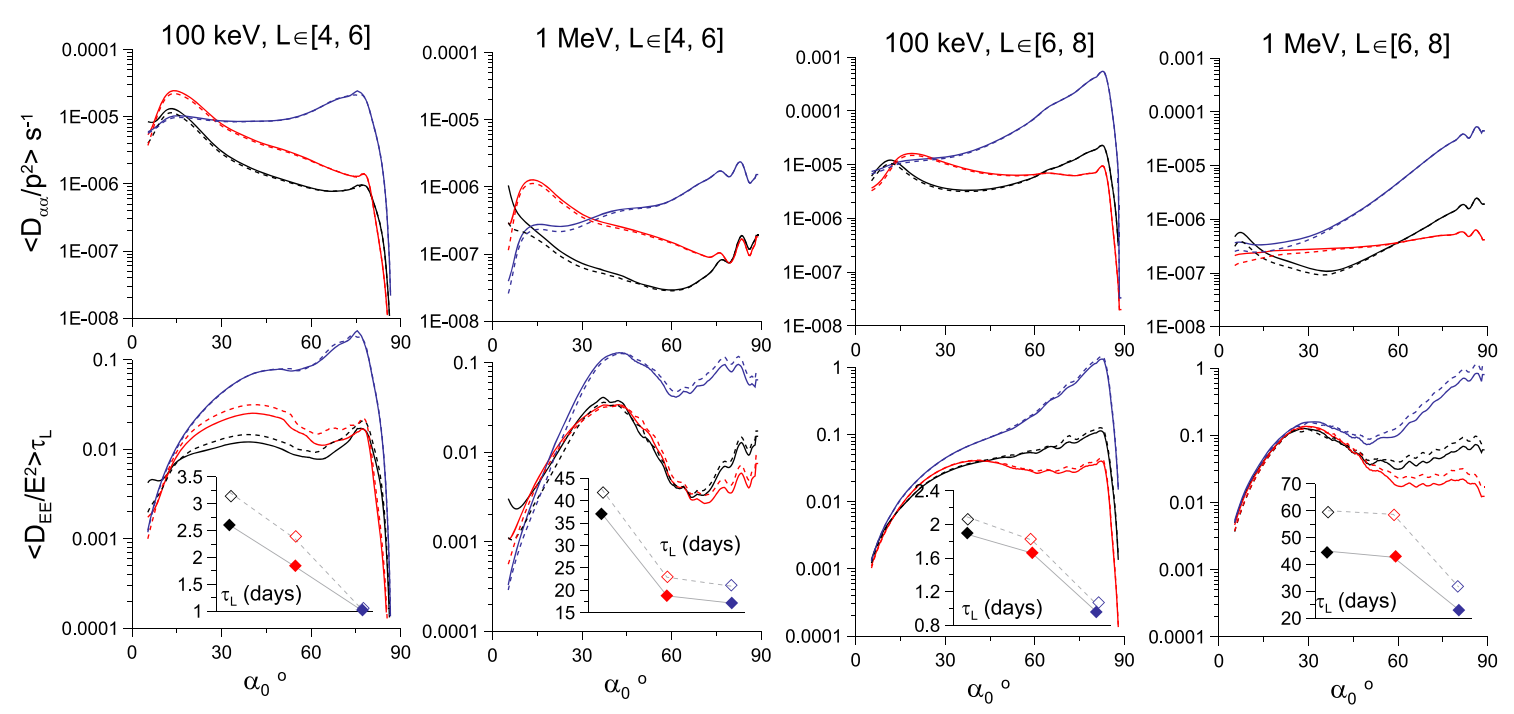

Figure 3. (top) MLT-averaged and bounce-averaged pitch angle diffusion coefficients for three Dst ranges and two energies for $L=4$ to 6 and $L=6$ to 8 . (bottom) $\left\langle D_{\mathrm{EE}} / E^{2}\right\rangle \tau_{L}$ as a function of $\alpha_{0}$ for three Dst ranges and two energies. Inserted panels show lifetimes $\tau_{L}$ in the three corresponding Dst ranges. Black, red, and blue curves correspond, respectively, to $|D s t|<10 \mathrm{nT}, D s t \in[-40,-10] \mathrm{nT}$, and $D s t \in[-80,-40] \mathrm{nT}$. The cases of constant (solid lines) and realistic latitudinally varying density (dashed lines) are considered.

corresponding case studies [Horne et al., 2005]. Nevertheless, it can be clearly seen in Figure 3 that $\tau_{L}\left\langle D_{\mathrm{EE}}\right\rangle_{B} / E^{2}$ increases tenfold during the most disturbed periods $D s t \in[-80,-40] \mathrm{nT}$ as compared to the intermediate range $D s t \in[-40,-10] \mathrm{nT}$. Poor Cluster statistics in the lower range Dst $<-100 \mathrm{nT}$ corresponding to the main phase of intense magnetic storms make it difficult to draw definite conclusions. Nevertheless, the upward trend of $\tau_{L}\left\langle D_{\mathrm{EE}}\right\rangle_{B} / E^{2}$ can be expected to continue (albeit at a probably slower rate) at lower $D s t$, as increased Landau damping by incoming suprathermal electrons further suppresses oblique waves and comparatively increases lifetimes.

In fact, $\tau_{L}\left\langle D_{\mathrm{EE}}\right\rangle_{B} / E^{2}$ would be independent of wave power (and thus of $D s t$ ) if the low-latitude to full bounce-average wave intensity ratio as well as the wave obliquity remained fixed. The large increase of $\tau_{L}\left\langle D_{\mathrm{EE}}\right\rangle_{B} / E^{2}$ with $|D s t|$ in Figure 3 stems from the stronger Landau damping of very oblique lower band chorus waves during the most active periods. The large wave power hike between $D s t \sim-25 \mathrm{nT}$ and Dst $\sim 60 \mathrm{nT}$ is almost fully compensated by the suppression of small amplitude very oblique waves (which are more efficient for pitch angle scattering than much higher amplitude parallel waves; see section 3), leading to only slightly reduced lifetimes and a much stronger electron energization when Dst $-40 \mathrm{nT}$ to $-80 \mathrm{nT}$ (i.e., during the early recovery phase of storms). Conversely, electron losses should increase during periods such that $D s t \in[-40,-10] \mathrm{nT}$ (corresponding for instance to isolated substorms or the late recovery phase of storms), while energization should remain the same as during quiet times ( $|D s t|<10 \mathrm{nT})$.

A characteristic time scale for electron acceleration is $\sim t_{\max } / 3$ from (8). In the loss-dominated regime of energization pertaining to the region $L \in[4,6]$, it yields between 10 and 1 day at $1 \mathrm{MeV}$ for $D s t \sim-50 \mathrm{nT}$ to $-100 \mathrm{nT}$, roughly consistent with observations [Horne et al., 2005; Fennell et al., 2012]. The corresponding average lifetimes vary between about 15 and 2 days, in relatively good agreement with previous realistic estimates [Shprits et al., 2008]. Slightly extrapolating the increase of chorus intensity observed over previous Dst ranges, the expected increased wave power for Dst $<-100 \mathrm{nT}$ would reduce $1 \mathrm{MeV}$ electron lifetimes to less than 1 day. Accordingly, important electron precipitations should occur during the main phase of storms. But electromagnetic ion cyclotron (EMIC) waves as well as outward radial diffusion induced by magnetopause shadowing are expected to be even more efficient than chorus in detrapping energetic electrons during the main phase of storms [Shprits et al., 2008; Turner et al., 2013; Shprits et al., 2013]. Then, losses should again prevail over acceleration, as it was already the case during moderately disturbed periods such that $D s t>-40 \mathrm{nT}$.

In the domain $L \in[6,8]$, diffusion rates and their behaviors are similar to the range $L \in[4,6]$, but for a decidedly smaller effect of very oblique waves, producing flattened profiles of $\left\langle D_{\alpha \alpha}\right\rangle_{B}$ for $D s t>-40 \mathrm{nT}$ (Figure 3). Comparing panels in Figure 1, oblique waves are indeed less intense at $L \in[6,8]$. This may be 
due to a slightly stronger Landau damping by plasma sheet electrons on the nightside and to a less effective refraction toward larger wave normal angles during propagation to higher latitudes in the presence of nonmonotonous variations of the geomagnetic field and cold plasma density on the dayside [e.g., see Roederer, 1970; Denton et al., 2006; Lakhina et al., 2010, and references therein]. The latter may also reduce chorus growth rates at low latitudes.

As a result of this mitigation of very oblique waves, electron lifetimes are similar or even slightly increased in the domain $L \in[6,8]$ as compared to $L \in[4,6]$, despite higher wave amplitudes at $\lambda>15^{\circ}$ on the dayside as well as on the nightside when $D s t<-40 \mathrm{nT}$. In turn, this leads to larger values of $\tau_{L}\left\langle D_{\mathrm{EE}}\right\rangle_{B} / E^{2}$ at $L \in[6,8]$, in general. It is especially true during more disturbed periods (Dst $\in[-80,-40] \mathrm{nT})$. Then, the large increase of near-equatorial chorus amplitudes on the nightside (as provided by CRRES) leads to $\tau_{L}\left\langle D_{\mathrm{EE}}\right\rangle_{B} / E^{2} \geq 1$ for $\alpha_{0}>65^{\circ}$ : the process of electron acceleration has entered a new regime of stronger, loss-independent energization (see section 3). Now, the typical acceleration time scale from (8) is $t_{\mathrm{max}} / 3 \sim E^{2} / 15\left\langle D_{\mathrm{EE}}\right\rangle_{B^{\prime}} 5$ times smaller than at $L \in[4,6]$ during the same active periods.

Finally, the effect of considering a more realistic latitude-varying cold plasma density [Denton et al., 2006] as compared to a constant one remains weak, in general. This effect is strongest for high-energy electrons $(E=1 \mathrm{MeV}$ ). Their lifetimes can be increased by about 30\% (see Figure 3).

\section{Interpretation of the Numerical Results and Discussion}

\subsection{Two Ways of Dealing With Disturbances: More Electron Losses or More Energization}

For the outer radiation belt, there appears to be two different ways of coping with incoming perturbations in the range $D s t \sim-2 \mathrm{nT}$ to $-80 \mathrm{nT}$. Moderate nightside electron injections can trigger parallel wave growth in nearly all MLT sectors, followed by refraction to larger wave normals during a relatively undamped propagation to high latitudes on the dayside, resulting finally in the precipitation of part of the incoming electrons into the ionosphere-effectively limiting trapped fluxes. But during more disturbed periods, more abundant plasma sheet suprathermal electrons can lead to a stronger Landau damping of oblique waves, comparatively reducing electron losses, while stronger (or more anisotropic) 30-50 keV electron injections produce more intense parallel waves at low latitudes and a much increased electron energization. Accordingly, the incoming energy of the perturbations is dispatched differently between wave and electron populations in the radiation belt. Let us discuss some of these points in more details below.

After many azimuthal orbits around the Earth, the statistical transfer of energy taking place between waves and particles should reach an asymptotic quasi-stable state. During periods of moderate geomagnetic activity (Dst $>-40 \mathrm{nT})$ at $L \in[4,6]$, a steep $1 / \sin \alpha_{0}$ increase of $\left\langle D_{\alpha \alpha}\right\rangle_{B}$ toward small pitch angles occurs in both the day and night sectors (see Figures 2 and 3), as a consequence of increased pitch angle scattering by very oblique waves there. As a result, a stronger downward phase space density (PSD) gradient should develop toward the loss cone. Horne et al. [2005] have suggested that $\sim 30 \mathrm{keV}$ injected electrons could generate parallel chorus waves via their efficient pitch angle scattering toward the loss cone. If the PSD gradient toward the loss cone is actually increased by efficient very oblique wave scattering, it is likely that (linear or quasilinear) wave growth should also be amplified as a result. This could partly account for the rather steep wave growth observed in the day sector between $\lambda \sim 0^{\circ}$ and $\lambda \sim 25^{\circ}$ [see Agapitov et al., 2013]. The possible existence of such a mechanism of quasi-linear parallel wave growth is discussed below in section 5.3.

During more disturbed periods $(-80 \mathrm{nT}<$ Dst $<-40 \mathrm{nT})$ at $L \in[4,8]$, the shape of the total pitch angle diffusion rate $\left\langle D_{\alpha \alpha}\right\rangle_{B}$ is actually reversed, now decreasing toward small $\alpha_{0}$, and lifetimes are only slightly diminished in spite of the much higher wave intensities. The PSD gradient toward small $\alpha_{0}$ should then be somewhat smoother than for Dst $\sim-25 \mathrm{nT}$, although drift shell splitting may also have increased slightly. Simultaneously, the total $\left\langle D_{\mathrm{EE}}\right\rangle_{B} / E^{2}$ has been multiplied more than tenfold at $\alpha_{0}>45^{\circ}$, much more than at $\alpha_{0}<45^{\circ}$ (see Figure 3). All these facts should lead to a better balance between acceleration and losses or even to a prevalence of electron acceleration over losses during such active periods.

In summary, it seems that when considering increasing geomagnetic disturbances (and associated electron injections), wave growth, subsequent wave refraction to large wave normal angles, and related particle losses are first favored, until disturbances/injections become strong enough to produce a large increase of wave intensity at lower latitudes in the night sector and a suppression of oblique waves at high latitudes on the dayside: the conjunction of these two events actually kick-starts a new regime, where electron energization now concurs efficiently with wave growth and scattering of particles toward the loss cone in absorbing 
the energy of incoming particle fluxes. These two regimes correspond roughly to Dst ranges located above and below $-40 \mathrm{nT}$.

What can satellite observations actually tell us about these different regimes? Clear cases of electron energization by chorus waves during disturbed periods such that $-80 \mathrm{nT}<D s t<-40 \mathrm{nT}$ have already been documented [e.g., see Shprits et al., 2006]. Some studies even suggest that the opposite range Dst $>-40 \mathrm{nT}$ may really be (statistically) much less prone to electron acceleration [Miyoshi et al., 2013; Zhao and Li, 2013], in line with the present work. The fact that weaker storms were not as well reproduced as strong storms by recent simulations considering only weakly oblique chorus waves [Kim et al., 2012] could also be an indication of the importance of oblique waves during weaker disturbances. However, many more detailed comparisons would be necessary to assess the importance/reality of the relevant processes.

\subsection{Two Preferred Regions for Electron Acceleration and the Variability of Energization}

For a typical trough density $n_{e} \propto 1 / L^{4}$ and a nearly constant chorus wave obliquity, equations (2), (4), and (5) can be combined to show that the most crucial quantity $\tau_{L}\left\langle D_{\mathrm{EE}}\right\rangle_{B} / E^{2}$ should vary like $1 / L^{2}$ to $1 / L^{3 / 2}$ from low (Dst $>-40 \mathrm{nT}$ ) to high (Dst <-40 nT) geomagnetic activity when considering a roughly constant wave intensity over $\lambda \sim 15^{\circ}-35^{\circ}$. As a result, a first peak of electron energization should be located near the lowest $L$ shell of the trough, just outside of the plasmapause (provided that plasma sheet energetic particle injections can readily arrive there). This is a fundamental point with far-reaching consequences on the radiation belt dynamics. Moreover, it is in very good agreement with the observed location $L_{\max }$ of the peak of MeV electron flux in the aftermath of storms [see Fennell et al., 2012; Zhao and Li, 2013, and references therein] which is roughly given by $L_{\max } \sim 1.25 L_{\mathrm{pp}}(D s t)$, where plasmapause position $L_{\mathrm{pp}}(D s t)$ is provided in the work by O'Brien and Moldwin [2003].

Nevertheless, we have already noted that lower band chorus wave obliquity decreases significantly above $L \sim 6$ (e.g., see section 2). As discussed above, such a decrease leads to an important increase of $\tau_{L}\left\langle D_{\mathrm{EE}}\right\rangle_{B} / E^{2}$. Consequently, electron acceleration is expected to rise around $L \sim 6$ too. During highly disturbed periods (Dst $<-50 \mathrm{nT}$ ) at $L \geq 6$, one even enters a regime of stronger energization independent of losses, where acceleration scales roughly like $B_{w}^{2}(L)$. Combined with magnetopause shadowing and radial diffusion, this enhanced energization could explain the statistical maximum of $\mathrm{MeV}$ electron flux observed around $L=$ 5.5-6 [Chen et al., 2007; Shprits et al., 2012]. To check the preceding conjectures, the variation of $\tau_{L}\left\langle D_{\mathrm{EE}}\right\rangle_{B} / E^{2}$ (i.e., of energy diffusion) with $L$ has been calculated numerically and plotted in Figure 4 for constant $(\mu, J)$ values (where $\mu$ and $J$ are the first and second adiabatic invariants), using realistic wave normal angle and wave amplitude distributions from Cluster for Dst $=-40$ to $-80 \mathrm{nT}$, as well as realistic density variations along field lines and as a function of $L$ [Sheeley et al., 2001; Denton et al., 2006]. Figure 4 shows that there are generally two peaks of energy diffusion, one located near the minimum $L$ value of the outer region close to the plasmapause (it is assumed that $L_{\mathrm{pp}} \leq 4$ in this figure) and a second one near $L=6$. However, the second maximum at $L \sim 6$ is generally sensibly higher and wider than the first one. It could explain why some recent statistical studies found only one global maximum at $L \sim 5.5-6$ [e.g., see Shprits et al., 2012].

However, since two peaks of electron energization are expected to occur at $L \sim 6$ and at $L \sim 1.25 L_{\mathrm{pp}}$, a double-belt structure of the outer radiation belt should not be uncommon during highly disturbed periods (such that $L_{p p}(D s t)<4$ ). Such a splitting of the outer belt has reportedly occurred during the recovery phase of at least eight storms in 1994-2003 in relation with local electron acceleration [Yuan and Zong, 2013]. Nonetheless, some of these double peaks could also be due to the remnant of a previous, larger belt, as noticed in other recent works [Turner et al., 2013; Shprits et al., 2013].

Significant plasma density structures are frequently observed outside of the plasmasphere as well as inside it (especially in the aftermath of storms). The presence of such kinds of density structures is likely to impact the above-described picture of electron energization and loss through the variation of $\tau_{L}\left\langle D_{\mathrm{EE}}\right\rangle_{B} / E^{2} \propto 1 / n_{e}$ : important density troughs might correspond to much higher energization of trapped electrons. The cold plasma density structure of the magnetosphere at the time when energization takes place could therefore translate into a (reversed) structuring of the population of energetic electrons trapped in Earth's radiation belts.

However, understanding the complex evolution of the outer radiation belt requires to explain in addition why similar magnetic storms induce different levels of global energization or losses, as first noted by Reeves et al. [2003]. We believe that one likely cause, if not the sole one, can be the observed variability of lower 


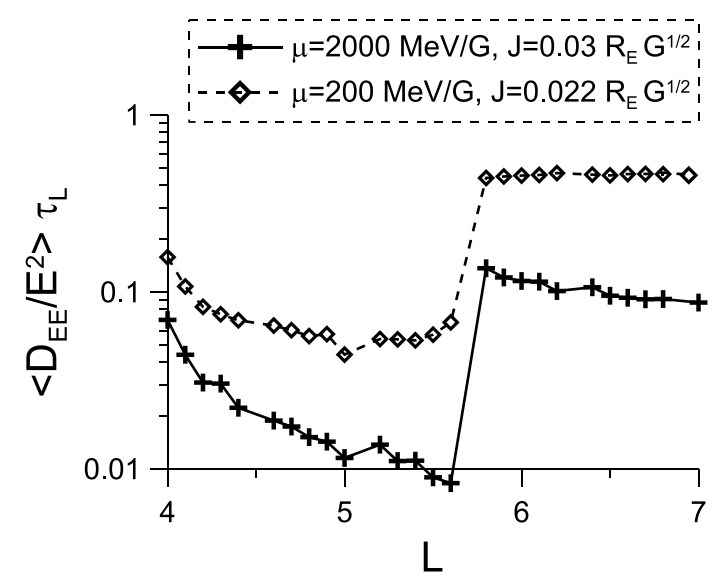

Figure 4. $\left\langle D_{\mathrm{EE}} / E^{2}\right\rangle \tau_{L}$ as a function of $L=4-7$ when $D s t=-40$ to $-80 \mathrm{nT}$, for constant first and second adiabatic invariants $\mu$ and $J$ such that $E \sim 2$ and $0.4 \mathrm{MeV}$ and $\alpha_{0} \sim 75^{\circ}$ at $L=4$. A realistic latitudinally varying density has been considered.

to $-10 \mathrm{nT}$, should produce much less energization than a slower initial recovery in the range $D s t=-80 \mathrm{nT}$ to $-40 \mathrm{nT}$ followed by a quick return back to $D s t \sim 0$.

However, the local behavior must still be averaged over the full outer belt to obtain a global picture of the increase or decrease of the trapped population. Moreover, additional mechanisms can be simultaneously at work and therefore need to be considered too: radial diffusion, adiabatic convection, and resonant interaction with other kinds of waves, such as fast magnetosonic waves [Horne et al., 2000; Mourenas et al., 2013] and especially EMIC waves during the storm's main phase [Turner et al., 2013]. Obviously, such a complicated task can only be addressed by means of full numerical simulations of the whole radiation belt [Shprits et al., 2006; Horne et al., 2013; Turner et al., 2013].

\subsection{The Possibility of Dayside Quasi-Linear Wave Growth}

Now, let us examine a potential mechanism of quasi-linear wave growth by deceleration of resonant 30-60 keV plasma sheet electrons in the day sector of the magnetosphere during moderately disturbed periods. When looking at quasi-linear energy diffusion rates, a few points are worthy of note. (i) First-order $(n=-1)$ cyclotron resonance with quasi-parallel chorus waves is the principal and almost the sole contributor to energy diffusion in the range $15^{\circ}<\alpha_{0}<45^{\circ}$ on the dayside (see Figure 5), other contributions by higher-order resonances or from very oblique waves being mostly negligible. (ii) Energy diffusion of 30-50 keV electrons such that $\alpha_{0}<45^{\circ}$ occurs at low latitudes $\lambda \sim 7^{\circ}-20^{\circ}$ generally distinct from latitudes where resonance with very oblique waves exists (see Figure 5). Thus, energy diffusion of 30-50 keV electrons can be simply modeled by (only) first-order cyclotron resonance with nearly parallel waves taking place over a narrow range $\Delta \lambda \leq 10^{\circ}$ of low latitudes.

In this specific case, the results of previous simplified studies of energy diffusion by purely parallel waves at a given (equatorial) latitude should roughly apply. The latter studies showed that such stochastic particle diffusion should occur preferentially toward regions of smaller electron phase space density (PSD) along so-called resonant diffusion characteristics at a given latitude [Summers et al., 1998]. Horne and Thorne [2003] suggested that energy diffusion by parallel waves near the equator should mainly lead to electron acceleration for $\alpha_{0}>50^{\circ}$ (due to a lower PSD at higher energy), while it could also lead to electron deceleration for $\alpha_{0}<45^{\circ}$ in case of a PSD decreasing steeply enough toward the loss cone. Fokker-Planck simulations including pitch angle and energy diffusion by parallel chorus waves have confirmed that such a scenario could really occur, even after bounce averaging over a finite range of low latitudes and even for broadband waves [Fu et al., 2012].

Nevertheless, mixed (energy and pitch angle) diffusion has frequently been found to mitigate energy diffusion by weakly oblique waves. A significantly reduced PSD gradient toward the loss cone in the asymptotic stable state may also suppress quasi-linear electron deceleration and corresponding wave growth. In the present study, however, we are dealing with a novel situation, where additional very oblique chorus waves 

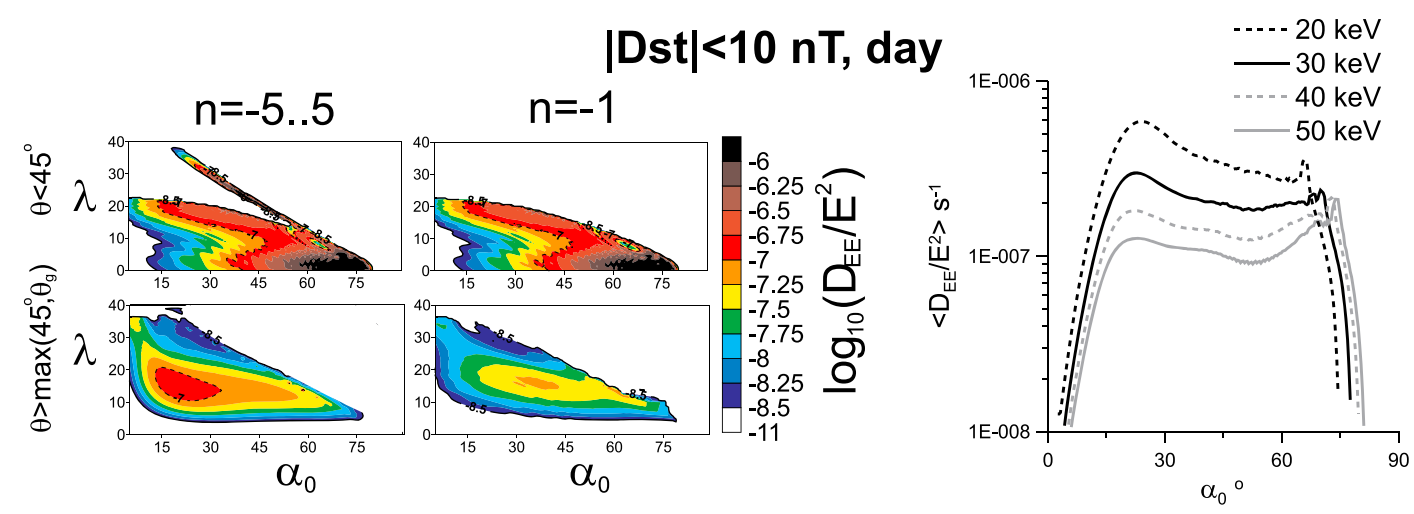

Figure 5. (left) Maps of local quasi-linear energy diffusion coefficients (considering either the sum of diffusion coefficients for $n=-5$ to +5 resonances or only the $n=-1$ cyclotron resonance) as a function of latitude and $\alpha_{0}$ for low geomagnetic activity in the day sector for $L \sim 4$ to 6 and $E=40 \mathrm{keV}$. (right) Dayside bounce-averaged energy diffusion rate for $E=20-50 \mathrm{keV}$ in the same conditions. A nearly constant plasma density is assumed.

are also present. This contrasts with past simulations, which considered only moderately oblique waves. Although very oblique waves are inefficient at scattering electrons in energy, they are extremely efficient in diffusing them in pitch angle toward the loss cone, much more than parallel waves (see Figures 2 and 3 and the works by Artemyev et al. [2012a], Mourenas et al. [2012b], and Artemyev et al. [2013b, 2013a]). The additional presence of very oblique waves should therefore steepen the downward PSD gradient toward the loss cone and consequently boost quasi-linear diffusion of particles in this direction, amplifying wave growth. Moreover, mixed diffusion is expected to be reduced in the case of a wide $\theta$ distribution as compared to the classical case of quasi-parallel waves [Albert, 2009]. Thus, the actual presence of very oblique waves during moderately disturbed periods should a priori increase quasi-linear electron deceleration toward the loss cone as compared to previous simulations dealing only with weakly oblique chorus waves. As a result, the proposed mechanism of quasi-linear wave growth induced by anisotropic $30 \mathrm{keV}$ electrons may turn out to play an important role on the dayside.

This mechanism can be further examined through simplified first-order analytical estimates. The maximum time $\Delta t_{\text {esc }}$ spent by the waves during one typical travel from the equator up to $\lambda \approx 20^{\circ}-25^{\circ}$ can be estimated as $\Delta t_{\mathrm{esc}} \approx 2 L R_{E} / 3 v_{g \| 0}=L R_{E} \Omega_{\text {peo }} /\left(3 c \sqrt{\omega_{m} \Omega_{\mathrm{ce} 0}}\right)$, where $v_{g \|}$ is the wave group velocity component parallel to the field line, $v_{g \| 0}$ is $v_{g \|}$ evaluated at $\theta=0, \lambda=0$, and $R_{E}$ is the Earth's radius. As discussed above, in the presence of a steep enough downward PSD gradient toward small $\alpha_{0}$, quasi-linear diffusion of electrons toward the loss cone by quasi-parallel chorus waves should correspond to an energy transfer from the particles to the waves [Summers et al., 1998; Horne and Thorne, 2003; Mourenas et al., 2012a]. Fundamentally, quasi-linear electron diffusion [Shapiro and Sagdeev, 1997] consists of a succession of small stochastic scatterings occuring in the two opposite directions along the curved diffusion characteristics, with varying magnitudes. For a wave experiencing many scatterings of electrons of various $\alpha_{0}$, it is very reminiscent of a random walk (Brownian-Wiener) process. To first order, the energy transferred to the wave should therefore vary like $E^{2} \approx 2 D_{\mathrm{EE}} t$, giving $\Delta E \sim D_{\mathrm{EE}} \Delta t / E \sim\left(D_{\mathrm{EE}} / E^{2}\right) E \Delta t$. Accordingly, the electron energy density transferred to the waves per unit time can be expressed as $n_{\text {ener }}\langle E\rangle\left\langle D_{\mathrm{EE}}\right\rangle / E^{2}$, where $n_{\text {ener }}$ is the density of electrons of average energy $\langle E\rangle$.

Energy diffusion rates for $E \sim 30 \mathrm{keV}$ have a wide maximum at $\alpha_{0}=15^{\circ}-70^{\circ}$ (see Figure 5 (right)). But Figure 5 also shows that for $E \leq 30 \mathrm{keV}$, the integral of $\left\langle D_{\mathrm{EE}} / E^{2}\right\rangle_{B}$ between $\alpha_{0} \sim 15^{\circ}$ and $45^{\circ}$ is twice larger than the same integral between $50^{\circ}$ and $70^{\circ}$. Thus, $E \sim 20-30 \mathrm{keV}$ electron deceleration (for $\alpha_{0}<45^{\circ}$ ) should be roughly twice more important than acceleration $\left(\alpha_{0}>50^{\circ}\right)$ when considering a typical electron distribution [Summers et al., 2009] $f\left(\alpha_{0}\right) \sim \sin \alpha_{0}$. Moreover, Figure 5 (left) shows that quasi-linear wave growth [i.e., $\left.D_{\mathrm{EE}}\left(\alpha_{0}<45^{\circ}\right)\right]$ should dominate at $\lambda \sim 10^{\circ}-25^{\circ}$, while quasi-linear wave damping $\left[D_{\mathrm{EE}}\left(\alpha_{0}>50^{\circ}\right)\right]$ should prevail at $\lambda<10^{\circ}$.

However, the usual linear wave growth [Kennel and Petschek, 1966] should also be present at very low latitudes. It could easily counterbalance quasi-linear damping in this region. At higher latitudes, geomagnetic field inhomogeneity and an increasing wave normal angle during propagation [Agapitov et al., 2013; Chen et al., 2013] are likely to pare down linear gain. Moreover, chorus consists of a wide ensemble of uncorrelated 
narrowband waves of latitude-varying frequencies. When waves are sufficiently intense or proximate in frequency to satisfy the Chirikov criterion (resonance overlap) for stochastic motion, one enters a regime of quasi-linear diffusion [Shapiro and Sagdeev, 1997; Tao et al., 2011] where frequency broadening due to randomization of particle trajectories should reduce linear wave growth [Dupree, 1966]. Such a stochastization of electron motion is actually enhanced by geomagnetic field inhomogeneity [Shklyar, 1981; Albert, 1993]. Quasi-linear wave growth should therefore progressively take over at latitudes $\lambda>5^{\circ}$ where dayside average amplitudes are already elevated ( $>4 \mathrm{pT}$ ), while any quasi-linear damping should probably be offset by linear growth at lower latitudes.

Accordingly, assuming that quasi-linear diffusion of $\sim 30 \mathrm{keV}$ electrons interacting resonantly with parallel chorus waves leads mainly to their deceleration, the average energy density gained by the waves per unit time can be written approximately as

$$
\frac{\Delta\left\langle B_{w}^{2}\right\rangle}{8 \pi \Delta t} \approx \frac{\left\langle D_{\mathrm{EE}}\right\rangle_{B}}{E^{2}} n_{\text {ener }}\langle E\rangle-v_{L} \frac{\left\langle B_{w}^{2}\right\rangle}{8 \pi} .
$$

The second term on the right-hand side of equation (9) contains the Landau damping $v_{L}$, assumed much smaller than the first term on the dayside for nearly quiet conditions. In this simple model, the maximum interval of time available for wave growth is $\Delta t_{\mathrm{esc}}$. For $\left\langle D_{\mathrm{EE}}\right\rangle_{B}$, a convenient first-order approximation is $\left\langle D_{\mathrm{EE}}\right\rangle_{B, \alpha_{0}=30^{\circ}}$ from equation (6), which was checked to give values in good agreement with numerical results in Figures 2 and 5 . Since $\left\langle D_{\mathrm{EE}}\right\rangle_{B}$ is proportional to $\left\langle B_{w}^{2}\right\rangle$, it follows that wave intensity $\left\langle B_{w}^{2}\right\rangle$ grows in time like $\sim \exp (G)$ with $G \propto n_{\text {ener }} t$. For large enough electron fluxes, quasi-linear diffusion could therefore lead to a wave increase akin to the usual exponential growth observed in the linear regime, albeit under a different form.

\subsection{Potential Limits on Trapped Fluxes Corresponding to Quasi-Linear Wave Growth}

Kennel and Petschek [1966] have demonstrated that anisotropic trapped electron fluxes can be bounded due to linearly self-generated whistler waves. Since waves grow exponentially with fluxes, any small increase in fluxes leads in turn to much stronger precipitations, until an effective self-limitation of fluxes finally occurs. A marginally stable state can be achieved when losses roughly balance incoming fluxes from the plasma sheet. More recently, Summers et al. [2009] extended this formulation to the relativistic regime, considering an ad hoc gain upper bound $G=3$ assumed to correspond to wave intensities high enough for quickly precipitating particles. But Kennel and Petschek [1966] did also assume that quasi-linear energy diffusion was negligible. While this was true for hiss waves, it is not true anymore for chorus. Moreover, actual flux limits should be smaller than either limit calculated for linear or quasi-linear wave growth alone. Therefore, it is interesting to estimate the flux limits corresponding to quasi-linear growth.

The quasi-linear wave intensity gain can be estimated from the ratio between the peak and minimum measured wave power, i.e., $G \sim \ln \left(B_{w}^{2}\left(\lambda=20^{\circ}\right) / B_{w}^{2}\left(\lambda=0^{\circ}\right)\right)$. Typically, these gains range from 1.5 at $L \in[4,6]$ to 1 at $L \in[6,8]$ during moderately disturbed periods. During stronger disturbances ( $D s t<-40 \mathrm{nT}$ ), gains are increased and reach 2.5 at all $L$ shells. Making use of equation (9) with time estimate $\Delta t \sim \Delta t_{\text {esc }}$ one obtains an upper limit on the density of quasi-stably trapped energetic electrons:

$$
n_{\mathrm{ener}}^{\max } \approx \frac{3 G v_{g \| 0} B_{w}^{2}}{16 \pi\langle E\rangle L R_{E}\left\langle D_{\mathrm{EE}}\right\rangle_{B} / E^{2}},
$$

where $\left\langle D_{\mathrm{EE}}\right\rangle_{B}$ is evaluated at $\alpha_{0} \approx 30^{\circ}$. Writing the stably trapped flux under the simplified form $F=n_{\text {ener }} V /$ $\left(S T_{b}\right) \sim n_{\text {ener }} V / 4$, where $V$ and $S$ are the flux tube volume and equatorial section, $T_{b}$ the electron bounce period, and $v=p c / \gamma$ the average electron velocity, one gets the maximum flux for a given $\langle E\rangle$ :

$$
F_{\max }\left[\mathrm{e} / \mathrm{cm}^{2} / \mathrm{s}\right] \approx \frac{1.8 \cdot 10^{10} G\langle E[\mathrm{MeV}]\rangle^{2 / 9}\left(\Omega_{p e 0} / \Omega_{\mathrm{ce} 0}\right)^{4 / 9}}{L^{4}(1+\langle E[\mathrm{MeV}]\rangle)^{7 / 9}\left(\omega_{m} / \Omega_{\mathrm{ce} 0}\right)^{7 / 9}} .
$$

Note that equation (11) should be valid only for $20 \mathrm{keV}<E<50 \mathrm{keV}$ in the outer belt (for cyclotron resonance to exist and electron acceleration to remain smaller than deceleration). But even in this range, $F_{\max }$ should only be accurate to within a factor 2 or 3 .

For a typical trough density $n_{e} \propto 1 / L^{4}$ and a nearly constant ratio $\omega_{m} / \Omega_{\text {ceo }} \sim 0.35$, one gets $F_{\max } \sim 2.5$. $10^{10} \mathrm{G}(L) / L^{7 / 2} \mathrm{e} / \mathrm{cm}^{2} / \mathrm{s}$, similar to the $1 / L^{4}$ scaling of the usual Kennel-Petschek limit. Making use of average 
chorus wave amplitudes measured by Cluster, one obtains $F_{\max } \sim 10^{8}(5 / L)^{7 / 2} \mathrm{e} / \mathrm{cm}^{2} / \mathrm{s}$ at $L \in[4,6]$ and $F_{\max } \sim 2 \cdot 10^{7}(7 / L)^{7 / 2} \mathrm{e} / \mathrm{cm}^{2} / \mathrm{s}$ at $L \in[6,8]$ for $\langle E\rangle \sim 30-50 \mathrm{keV}$. These values compare well with actual measurements of omnidirectional time-averaged fluxes provided in the AE8 model [Vette, 1991] at $L=4.5$ to 7 . Of course, $F_{\max }$ represents only a rough upper limit on average fluxes: very strong injections may drive fluxes to higher levels corresponding to higher chorus wave intensities and gains. Besides, it is worth noting that in the limit $\omega_{m} / \Omega_{\text {ceo }} \ll 1$, the limiting flux $F_{\max }$ given by equation (11) becomes exceedingly large as quasi-linear energy diffusion vanishes, in agreement with estimates from Kennel and Petschek [1966]. In such a case, the Kennel-Petschek (linear gain) flux limit must be used alone. Finally, since it has been shown above that quasi-linear wave growth could potentially play an important role in controlling actual fluxes of injected plasma sheet electrons, full-scale Fokker-Planck numerical simulations would be welcomed in the future to assess more accurately the importance of this mechanism. But this is beyond the scope of the present paper.

\section{Conclusions}

Full statistics of lower band chorus wave distributions (amplitudes and obliquity) as functions of $L \in[4,8]$, latitude, MLT, and geomagnetic activity range have been obtained from 10 years of measurements on board the spacecraft Cluster. Based on an analysis of these results and numerical calculations of corresponding quasi-linear diffusion rates and lifetimes, it has been shown that

1. Dayside very oblique lower band chorus waves $\left(\theta>60^{\circ}\right)$ dominate pitch angle diffusion of energetic electrons for Dst $\geq-40 \mathrm{nT}$ at $L \in[4,8]$ (although their average amplitude is smaller than parallel wave amplitudes). Their influence decreases during more disturbed periods, probably in relation with stronger Landau damping by plasma sheet suprathermal electrons. Moreover, oblique waves in the very close vicinity of the resonance cone angle (likely the most damped) are not essential in diffusion rate calculations at high energy $E>0.3 \mathrm{MeV}$.

2. Nightside wave growth is confined to lower latitudes and smaller wave obliquity, probably due to higher Landau damping by suprathermal electrons which are injected in this sector.

3. Electron lifetimes decrease unexpectedly slowly with decreasing $D s t$, because the increase of the wave intensity is almost compensated by a decrease of wave obliquity.

4. Electron energization by chorus waves occurs in a loss-dominated regime for $L \in[4,6]$ and $D s t>-80 \mathrm{nT}$ as well as for $L \in[6,8]$ and $D s t>-40 \mathrm{nT}$. Only during the most disturbed periods at $L \geq 6$ does a stronger energization occur independently of losses.

5. Two peaks of energization should occur, one just outside the plasmapause and a second one near $L \sim 6$.

6. The variability of lower band chorus wave obliquity with both geomagnetic activity and MLT, related to the strength of suprathermal electron injections, probably accounts for part of the observed variability of the degree of energization and loss in the outer radiation belt.

7. Lower band chorus wave growth between the equator and $\lambda \sim 25^{\circ}$ on the dayside when Dst $\geq-40 \mathrm{nT}$ could be partly the result of quasi-linear pitch angle and energy diffusion of $20-50 \mathrm{keV}$ electrons, oblique wave pitch angle scattering increasing the electron PSD gradient and favoring energy diffusion by parallel waves toward the loss cone in the day sector.

8. Chorus wave growth due to quasi-linear energy diffusion by parallel waves could impose another upper limit on quasi-stably trapped 20-50 keV electron fluxes coming from the plasma sheet.

As a next step, the important distribution of wave obliquity measured by Cluster should be cross checked and improved when new data statistics from more recent spacecraft missions such as the Van Allen Probes become available. Furthermore, numerical ray tracing calculations of chorus wave propagation including full thermal effects (damping) based on actual (measured) electron distributions will be necessary to assess more precisely the probability of presence of waves in the close vicinity of the resonance cone. As concerns radiation belt electron energization, numerically solving the full Fokker-Planck diffusion equation will be required to investigate the different regimes in more details. Finally, linear, quasi-linear, and nonlinear wave growths should all be considered, as well as the quasi-coherent property of part of the chorus wave packets, which should likely make the full picture of chorus wave-electron interactions even more complex than it looks now. 


\section{Appendix A: Thermal Effects on Oblique Chorus Quasi-Linear Diffusion Rates}

Although the assumption of a cold plasma whistler mode dispersion has been used in this paper, previous works have shown that it can break down for wave normal angles very close to the resonance cone [Hashimoto et al., 1977; Horne and Sazhin, 1990], where the wave's parallel phase velocity $\omega / k_{\|}$becomes much smaller. This appendix is devoted to a short study of potential thermal (kinetic) effects on diffusion rates, based on approximate analytical expressions of the full dispersion equation. While looking at the full numerical (complex) solutions is certainly preferable, satellite measurements of the electron distribution in the magnetosphere show a sufficient range of variability of the relevant electron densities and temperatures to justify considering simpler approximate solutions as a first step.

Various satellite measurements [e.g., Horne and Sazhin, 1990; Horne et al., 2000; Bortnik et al., 2007; Li et al., 2010, and references therein] have shown that at $L \sim 5$ during moderately disturbed periods, the electron population consists primarily of a nearly $1 \mathrm{eV}$ cold component together with a roughly $100 \mathrm{eV}$ hot component. Moreover, hot electrons are usually much more abundant $(\approx 10$ times) on the nightside than on the dayside, because they are believed to arrive from the plasma sheet near the midnight sector and further drift slowly toward the noon sector while experiencing losses to the atmosphere [Li et al., 2010]. Thus, we assume a hot electron density around $1 \mathrm{~cm}^{-3}$ on the nightside and less than $0.1 \mathrm{~cm}^{-3}$ on the dayside at $L \sim 5$. At the equator, it gives electron $\beta$ values $\beta \sim 4 \cdot 10^{-5}$ in the day sector and $\beta \sim 3 \cdot 10^{-4}$ in the night sector for a cold plasma density such that $\Omega_{\mathrm{pe} 0} / \Omega_{\text {ce0 }} \sim 4.5$ in the trough [Sheeley et al., 2001]. Note that $\beta$ decreases with latitude since $\beta=\sum\left(\Omega_{\mathrm{pe}, i} / \Omega_{\mathrm{ce}}\right)^{2}\left(T_{e, i} / m_{e} c^{2}\right)$, where subscripts $i$ correspond to cold and hot components.

Full numerical calculations have shown that when finite temperature effects are included, whistler mode waves are able to propagate at angles $\theta$ above the resonance cone angle $\theta_{r}$ [Hashimoto et al., 1977]; in this domain, however, they are experiencing a strong Landau damping when a sufficient proportion of (hot) electrons reach Landau resonance $\left(\omega \sim k_{\|} v\right)$ with the waves [Horne and Sazhin, 1990]. Based on the numerical results of Horne and Sazhin [1990], in the presence of a small hot electron component with thermal velocity $v_{\text {hot }}$ which is 1000 to 40 times less dense than the cold plasma, a rough condition for moderate Landau damping of the considered oblique waves is $\omega / k_{\|}>v_{\text {hot }}$ to $1.8 v_{\text {hot }}$. For waves propagating near the resonance cone, the latter conditions are equivalent to requiring that the wave refractive index $N$ be smaller than $N_{\text {damped }}$ to $N_{\text {damped }} / 1.8$ with

$$
N_{\text {damped }} \sim \frac{\Omega_{\text {ce }}}{\omega} \sqrt{\frac{512}{2 T_{\text {hot }}[\mathrm{keV}]}} .
$$

Since the refractive index $N$ steeply increases near $\theta=\theta_{r}$, waves should be quickly damped above $\theta_{r}$ and should not be observed there, in general, allowing us to restrict our consideration to $\theta<\theta_{r}$. Moreover, our $\beta$ values are close to the cases studied in Figure 1 in the work by Hashimoto et al. [1977] (where $\beta=2 \cdot 10^{-5}$ ) and in Figure 1 in the work by Horne and Sazhin [1990] (where $\beta=1.5 \cdot 10^{-4}$ ). As noted by Hashimoto et al. [1977], for such low $\beta$ values the cold plasma whistler mode dispersion should be only slightly modified by thermal effects below $\theta_{r}$.

Consequently, a first-order approach for taking into account thermal effects at low $\beta$ is to keep on using the Appleton-Hartree cold plasma dispersion relation as long as the wave refractive index $N \leq$ $\min \left(300, N_{\text {thermal, } \theta_{r}}\right)$, where $N_{\text {thermal }}$ is the refractive index calculated from the quasi-electrostatic analytic expression including thermal effects derived by Horne and Sazhin [1990]. One simply stops integrating over $\theta$ when the cold-plasma $N(\theta)$ becomes larger than $N_{\text {thermal, } \theta_{r}}$. Considering here isotropic electron distributions and $\theta=\theta_{r}$, equations (2)-(5) in the work by Horne and Sazhin [1990] lead to $N_{\text {thermal }}\left(\theta_{r}\right) \sim\left(\Omega_{\text {pe }} / \omega\right) / \beta^{1 / 4}$, yielding finally

$$
N_{\text {thermal }, \theta_{r}} \sim \frac{\sqrt{\Omega_{\mathrm{pe}} \Omega_{\mathrm{ce}}}}{\omega} \frac{2^{1 / 4} 4}{\left[T_{\text {eff }}(\mathrm{keV})\right]^{1 / 4}},
$$

for $\Omega_{\text {pe }} / \omega \gg 1$ and $\omega / \Omega_{\text {ce }}<1 / 2$, where we can take an effective electron temperature $T_{\text {eff }} \sim 1 \mathrm{eV}$ and $10 \mathrm{eV}$ for dayside and nightside at $L \sim 5$, respectively. Using the above effective temperature, $N_{\text {thermal, } \theta_{r}}$ varies between 100 and 200 typically between the equator and high latitudes. It is worth noting that the upper bound $N_{\text {damped }}$ for low Landau damping varies between $\sim 1.7 N_{\text {thermal, } \theta_{r}}$ at $\lambda \sim 10^{\circ}$ and more than $2 N_{\text {thermal, } \theta_{r}}$ at $\lambda>25^{\circ}$, which justifies using only $N_{\text {thermal, } \theta_{r}}$ for the upper bound on $N$. Furthermore, we have checked 

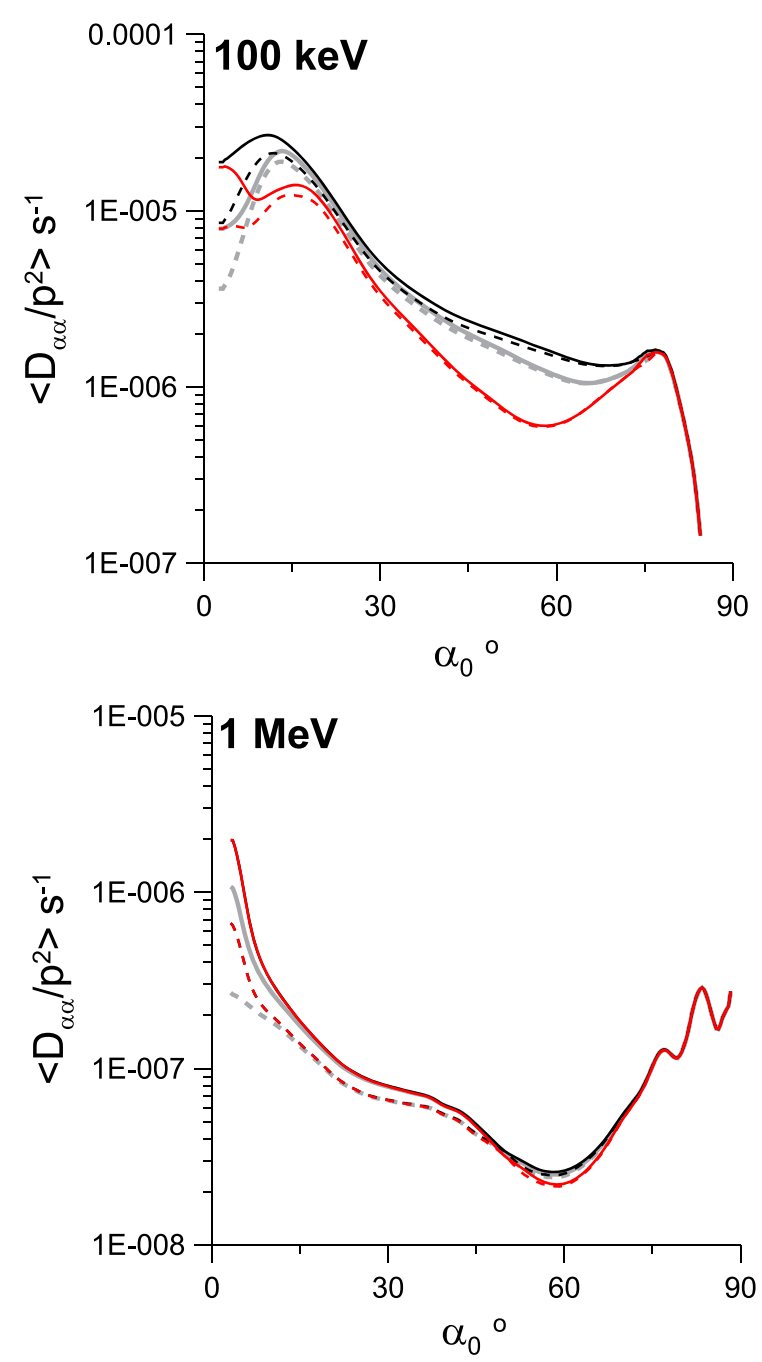

Figure A1. Bounce-averaged pitch angle diffusion coefficients for $|D s t|<10 \mathrm{nT}$ and two energies (day sector at $L \sim 5$ ). Grey curves show standard calculations (with the condition $N<N_{\text {max }}$, see text). Black curves show the scattering rates calculated only with the limit $N<300$, corresponding to a nearly null plasma temperature. Red curves represent the rates calculated for a condition $N<\min \left(300, N_{\text {thermal, } \theta_{r}}\right)$ corresponding to an effective temperature of $10 \mathrm{eV}$. In each case, one considers either a constant plasma density along field lines (solid lines) or a latitude-varying density (dashed lines). In the $1 \mathrm{MeV}$ case, black and red curves are nearly indistinguishable. latter case, higher-order resonances contribute only for waves much closer to the resonance cone angle. For $\alpha_{0}<15^{\circ}$, significant wave-particle coupling requires $\Omega_{\text {ce }}(\lambda) / \Omega_{\text {ce } 0} \geq\left(\omega / \Omega_{\text {ce0 }} \sin \alpha_{0}\right)^{2 / 3}$ and therefore higher latitudes $\lambda \geq 30^{\circ}$ [Mourenas et al., 2012b]. In this situation, $|n|\left(\theta_{g}\right)$ becomes smaller than 1 and only waves closer to $\theta_{r}$ can lead to high-order cyclotron resonance. Nonetheless, even in this case, lower energy requires $\theta$ values closer to $\theta_{r}$ than higher $E$.

Thus, oblique waves very close to the resonance cone angle $\theta_{r}$ become sensibly more important as electron energy is reduced [e.g., see Artemyev et al., 2013b, Figure 10]. In particular, one gets $|n|=10$ for $N \sim 35$ and 150 for $E=1 \mathrm{MeV}$ and $0.1 \mathrm{MeV}$, respectively, at $\lambda \sim 10^{\circ}$ for $\alpha_{0}>15^{\circ}$ (see Figure 5). Then, $N_{\text {thermal, } \theta_{r}} \sim 100$ for $T_{\text {eff }}=10 \mathrm{eV}$, so that $N<N_{\text {thermal, } \theta_{r}} / 2.5 \sim N_{\text {max }} / 3.5 \sim N_{\text {damped }} / 4$ for $E=1 \mathrm{MeV}$. However, at lower energy $E=0.1 \mathrm{MeV}$, one finds $N>N_{\text {thermal, } \theta_{r}} \sim N_{\max } / 1.5 \sim N_{\text {damped }} / 1.7$. At $\lambda \sim 30^{\circ}$ for $\alpha_{0} \sim 5-10^{\circ}$, one gets $|n|=10$ for $N \sim 200$ and 800 for $E=1 \mathrm{MeV}$ and $0.1 \mathrm{MeV}$, respectively. One has now $N_{\text {thermal, } \theta_{r}} \sim 200$ for $T_{\text {eff }}=10 \mathrm{eV}$ : 


\section{Acknowledgements}

This work was partly supported by CNES through the grant "Modeles d'ondes." We thank the ESA Cluster Active Archive for providing the STAFF-SA data set. Work of A.V.A. was also partially supported by the grant MK-1781.2014.2.

Michael Liemohn thanks the reviewer for assistance in evaluating this paper. while at $1 \mathrm{MeV}$ the needed $N$ is smaller than $N_{\text {thermal, } \theta_{r}} \sim N_{\text {max }} \sim N_{\text {damped }} / 2.5$, it becomes larger than all these bounds at $100 \mathrm{keV}$.

The above estimates suggest that thermal effects should induce relatively small changes in scattering rates for energetic electrons $(E \sim 1 \mathrm{MeV})$ as compared to our standard numerical calculations performed with the sole condition $N<N_{\text {max }}$. Numerical diffusion rates plotted in Figure A1 for the same parameters at $L \sim 5$ on the dayside confirm that it is indeed the case. The standard pitch angle diffusion rate (grey curves) is very close to the diffusion rate obtained for $T_{\text {eff }} \sim 10 \mathrm{eV}$ with the condition $N<\min \left(300, N_{\text {thermal, } \theta_{r}}\right.$ ) (red curves), which is itself almost identical to the null-temperature diffusion rate (black curves). The effect of a latitude-varying plasma density [Denton et al., 2006] is actually more important than thermal effects (at least in their rough implementation considered here) for $E=1 \mathrm{MeV}$ in Figure A1. Finally, numerical calculations displayed in Figure 3 demonstrate that corresponding lifetime modifications will remain small. For lower energy electrons ( $E \sim 100 \mathrm{keV})$, thermal effects are stronger as expected: the diffusion rate obtained for $T_{\text {eff }} \sim 10 \mathrm{eV}$ is now always sensibly smaller than for a null temperature plasma. Nevertheless, the difference between our standard calculations and these two limiting cases remains smaller than a factor of 2 . It implies only little changes in lifetimes on the dayside (for $T_{\text {eff }} \approx 1-3 \mathrm{eV}$ ). Since lifetimes are principally determined by the stronger dayside scattering (see Figure 2), thermal effects on azimuthal-drift-averaged lifetimes should remain small too.

Consequently, the (standard) diffusion rates calculated in the main body of this paper can be considered as approximately correct for $L \sim 4$ to 6 in the day sector. In the night sector, however, the tenfold denser hot electron component can still lead to a strong Landau damping for waves very close to $\theta_{r}$ [Horne and Sazhin, 1990]. Numerically solving the full dispersion equation would be needed to examine this point in the future. Nevertheless, very oblique waves are significantly more rare on the nightside (see section 2). Moreover, previous investigations have shown that a partial suppression of oblique waves at $\lambda>20^{\circ}$ should not strongly modify lifetimes, in general [Artemyev et al., 2013b]. At $L=6$ to 8 , very oblique waves are significantly less present (see section 2 ) while the hot electron component seems to be roughly similar at $L=6-7$ and 5-6 [ $L i$ et al., 2010], suggesting again rather weak thermal effects, especially when compared to density variation effects which may be expected to increase [e.g., see Denton et al., 2006].

\section{References}

Agapitov, O., V. Krasnoselskikh, Y. V. Khotyaintsev, and G. Rolland (2012), Correction to "A statistical study of the propagation characteristics of whistler waves observed by Cluster", Geophys. Res. Lett., 39, L24102, doi:10.1029/2012GL054320.

Agapitov, O., A. Artemyev, V. Krasnoselskikh, Y. V. Khotyaintsev, D. Mourenas, H. Breuillard, M. Balikhin, and G. Rolland (2013), Statistics of whistler-mode waves in the outer radiation belt: Cluster STAFF-SA measurements, J. Geophys. Res. Space Physics, 118, 3407-3420, doi:10.1002/jgra.50312.

Albert, J. M. (1993), Cyclotron resonance in an inhomogeneous magnetic field, Phys. Fluids B, 5, 2744-2750, doi:10.1063/1.860715.

Albert, J. M. (2005), Evaluation of quasi-linear diffusion coefficients for whistler mode waves in a plasma with arbitrary density ratio, $J$. Geophys. Res., 110, A03218, doi:10.1029/2004JA010844.

Albert, J. M. (2009), The coupling of quasi-linear pitch angle and energy diffusion, J. Atmos. Sol. Terr. Phys., 71, 1664-1668, doi:10.1016/j.jastp.2008.11.014

Albert, J. M., and Y. Y. Shprits (2009), Estimates of lifetimes against pitch angle diffusion, J. Atmos. Sol. Terr. Phys., 71, 1647-1652, doi:10.1016/j.jastp.2008.07.004.

Andronov, A. A., and V. Yu. Trakhtengerts (1964), Kinetic instability of the Earth's outer radiation belt, Geomagn. Aeron., 4, $233-242$.

Artemyev, A., O. Agapitov, H. Breuillard, V. Krasnoselskikh, and G. Rolland (2012a), Electron pitch-angle diffusion in radiation belts: The effects of whistler wave oblique propagation, Geophys. Res. Lett., 39, L08105, doi:10.1029/2012GL051393.

Artemyev, A., V. Krasnoselskikh, O. Agapitov, D. Mourenas, and G. Rolland (2012b), Non-diffusive resonant acceleration of electrons in the radiation belts, Phys. Plasmas, 19, 122901, doi:10.1063/1.4769726.

Artemyev, A., O. Agapitov, D. Mourenas, V. Krasnoselskikh, and L. Zelenyi (2013a), Storm-induced energization of radiation belt electrons: Effect of wave obliquity, Geophys. Res. Lett., 40, 4138-4143, doi:10.1002/grl.50837.

Artemyev, A., D. Mourenas, O. Agapitov, and V. Krasnoselskikh (2013b), Parametric validations of analytical lifetime estimates for radiation belt electron diffusion by whistler waves, Ann. Geophys., 31, 599-624, doi:10.5194/angeo-31-599-2013.

Bakhareva, M. F. (2005), Time variations in energetic particle fluxes at different types of statistical acceleration and the variation properties during geomagnetic disturbances, Geomagn. Aeron., 45, 551-561.

Balikhin, M. A., M. Gedalin, G. D. Reeves, R. J. Boynton, and S. A. Billings (2012), Time scaling of the electron flux increase at GEO: The local energy diffusion model vs observations, J. Geophys. Res., 117, A10208, doi:10.1029/2012JA018114.

Bortnik, J., R. M. Thorne, and N. P. Meredith (2007), Modeling the propagation characteristics of chorus using CRRES suprathermal electron fluxes, J. Geophys. Res., 112, A08204, doi:10.1029/2006JA012237.

Breuillard, H., Y. Zaliznyak, V. Krasnoselskikh, O. Agapitov, A. Artemyev, and G. Rolland (2012), Chorus wave-normal statistics in the Earth's radiation belts from ray tracing technique, Ann. Geophys., 30, 1223-1233.

Bunch, N. L., M. Spasojevic, Y. Y. Shprits, X. Gu, and F. Foust (2013), The spectral extent of chorus in the off-equatorial magnetosphere, J. Geophys. Res. Space Physics, 118, 1700-1705, doi:10.1029/2012JA018182.

Carpenter, D. L., and R. R. Anderson (1992), An ISEE/whistler model of the equatorial electron density in the magnetosphere, J. Geophys. Res., 97, 1097-1108, doi:10.1029/91JA01548. 
Cattell, C., et al. (2008), Discovery of very large amplitude whistler-mode waves in Earth's radiation belts, Geophys. Res. Lett., 35, L01105, doi:10.1029/2007GL032009.

Chen, Y., G. D. Reeves, and R. H. W. Friedel (2007), The energization of relativistic electrons in the outer Van Allen radiation belt, Nat. Phys., 3, 614-617, doi:10.1038/nphys655.

Chen, L., R. M. Thorne, W. Li, and J. Bortnik (2013), Modeling the wave normal distribution of chorus waves, J. Geophys. Res. Space Physics, 118, 1074-1088, doi:10.1029/2012JA018343.

Cornilleau-Wehrlin, N., et al. (2003), First results obtained by the Cluster STAFF experiment, Ann. Geophys., 21, 437-456, doi:10.5194/angeo-21-437-2003.

Denton, R. E., K. Takahashi, I. A. Galkin, P. A. Nsumei, X. Huang, B. W. Reinisch, R. R. Anderson, M. K. Sleeper, and W. J. Hughes (2006), Distribution of density along magnetospheric field lines, J. Geophys. Res., 111, A04213, doi:10.1029/2005JA011414.

Dupree, T. H. (1966), A perturbation theory for strong plasma turbulence, Phys. Fluids, 9, 1773-1782, doi:10.1063/1.1761932.

Fennell, J. F., S. Kanekal, and J. L. Roeder (2012), Storm responses of radiation belts during solar cycle 23: HEO satellite observations, in Dynamics of the Earth's Radiation Belts and Inner Magnetosphere, Geophys. Monogr. Ser., vol. 199, edited by D. Summers et al., pp. 371-384, AGU, Washington, D. C., doi:10.1029/2012GM001356.

Fu, H. S., J. B. Cao, Q.-G. Zong, H. Y. Lu, S. Y. Huang, X. H. Wei, and Y. D. Ma (2012), The role of electrons during chorus intensification: Energy source and energy loss, J. Atmos. Sol. Terr. Phys., 80, 37-47, doi:10.1016/j.jastp.2012.03.004.

Glauert, S. A., and R. B. Horne (2005), Calculation of pitch angle and energy diffusion coefficients with the PADIE code, J. Geophys. Res., 110, A04206, doi:10.1029/2004JA010851.

Hashimoto, K., I. Kimura, and H. Kumagai (1977), Estimation of electron temperature by VLF waves propagating in directions near the resonance cone, Planet. Space Sci., 25, 871-877.

Hayakawa, M., Y. Yamanaka, M. Parrot, and F. Lefeuvre (1984), The wave normals of magnetospheric chorus emissions observed on board GEOS 2, J. Geophys. Res., 89, 2811-2821, doi:10.1029/JA089iA05p02811.

Horne, R. B., and S. S. Sazhin (1990), Quasielectrostatic and electrostatic approximations for whistler mode waves in the magnetospheric plasma, Planet. Space Sci, 38(2), 311-318.

Horne, R. B., G. V. Wheeler, and H. S. C. K. Alleyne (2000), Proton and electron heating by radially propagating fast magnetosonic waves, J. Geophys. Res., 105, 27,597-27,610, doi:10.1029/2000JA000018.

Horne, R. B., and R. M. Thorne (2003), Relativistic electron acceleration and precipitation during resonant interactions with whistler-mode chorus, Geophys. Res. Lett., 30(10), 1527, doi:10.1029/2003GL016973.

Horne, R. B., R. M. Thorne, S. A. Glauert, J. M. Albert, N. P. Meredith, and R. R. Anderson (2005), Timescale for radiation belt electron acceleration by whistler mode chorus waves, J. Geophys. Res., 110, A03225, doi:10.1029/2004JA010811.

Horne, R. B., S. A. Glauert, N. P. Meredith, D. Boscher, V. Maget, D. Heynderickx, and D. Pitchford (2013), Space weather impacts on satellites and forecasting the Earth's electron radiation belts with SPACECAST, Space Weather, 11, 169-186, doi:10.1002/swe.20023.

Horne, R. B., T. Kersten, S. A. Glauert, N. P. Meredith, D. Boscher, A. Sicard-Piet, R. M. Thorne, and W. Li (2013), A new diffusion matrix for whistler mode chorus waves, J. Geophys. Res. Space Physics, 118, 6302-6318, doi:10.1002/jgra.50594.

Kennel, C. F., and H. E. Petschek (1966), Limit on stably trapped particle fluxes, J. Geophys. Res., 71, 1-28.

Kim, K.-C., Y. Shprits, D. Subbotin, and B. Ni (2012), Relativistic radiation belt electron responses to GEM magnetic storms: Comparison of CRRES observations with 3-D VERB simulations, J. Geophys. Res., 117, A08221, doi:10.1029/2011JA017460.

Lakhina, G. S., B. T. Tsurutani, O. P. Verkhoglyadova, and J. S. Pickett (2010), Pitch angle transport of electrons due to cyclotron interactions with the coherent chorus subelements, J. Geophys. Res., 115, A00F15, doi:10.1029/2009JA014885.

Lauben, D. S., U. S. Inan, T. F. Bell, and D. A. Gurnett (2002), Source characteristics of ELF/VLF chorus, J. Geophys. Res., 107(A12), 1429, doi:10.1029/2000JA003019.

Lengyel-Frey, D., W. M. Farrell, R. G. Stone, A. Balogh, and R. Forsyth (1994), An analysis of whistler waves at interplanetary shocks, J. Geophys. Res., 99(A7), 13,325-13,334.

Li, W., R. M. Thorne, V. Angelopoulos, J. Bortnik, C. M. Cully, B. Ni, O. LeContel, A. Roux, U. Auster, and W. Magnes (2009), Global distribution of whistler-mode chorus waves observed on the THEMIS spacecraft, Geophys. Res. Lett., 36, L09104, doi:10.1029/2009GL037595.

Li, W., R. M. Thorne, J. Bortnik, Y. Nishimura, V. Angelopoulos, L. Chen, J. P. McFadden, and J. W. Bonnell (2010), Global distributions of suprathermal electrons observed on THEMIS and potential mechanisms for access into the plasmasphere, J. Geophys. Res., 115, A00J10, doi:10.1029/2010JA015687.

Li, W., J. Bortnik, R. M. Thorne, C. M. Cully, L. Chen, V. Angelopoulos, Y. Nishimura, J. B. Tao, J. W. Bonnell, and O. LeContel (2013), Characteristics of the Poynting flux and wave normal vectors of whistler-mode waves observed on THEMIS, J. Geophys. Res. Space Physics, 118, 1461-1471, doi:10.1002/jgra.50176.

Lyons, L. R. (1974), Pitch angle and energy diffusion coefficients from resonant interactions with ion-cyclotron and whistler waves, J. Plasma Phys., 12, 417-432, doi:10.1017/S002237780002537X.

Lyons, L. R., R. M. Thorne, and C. F. Kennel (1971), Electron pitch-angle diffusion driven by oblique whistler-mode turbulence, J. Plasma Phys., 6, 589-606, doi:10.1017/\$0022377800006310.

Lyons, L. R., R. M. Thorne, and C. F. Kennel (1972), Pitch-angle diffusion of radiation belt electrons within the plasmasphere, J. Geophys. Res., 77, 3455-3474, doi:10.1029/JA077i019p03455.

Means, J. D. (1972), Use of the three-dimensional covariance matrix in analyzing the polarization properties of plane waves, J. Geophys. Res., 77, 5551-5559, doi:10.1029/JA077i028p05551.

Meredith, N. P., R. B. Horne, A. Sicard-Piet, D. Boscher, K. H. Yearby, W. Li, and R. M. Thorne (2012), Global model of lower band and upper band chorus from multiple satellite observations, J. Geophys. Res., 117, A10225, doi:10.1029/2012JA017978.

Miyoshi, Y., R. Kataoka, Y. Kasahara, A. Kumamoto, T. Nagai, and M. F. Thomsen (2013), High-speed solar wind with southward interplanetary magnetic field causes relativistic electron flux enhancement of the outer radiation belt via enhanced condition of whistler waves, Geophys. Res. Lett., 40, 4520-4525, doi:10.1002/grl.50916.

Mourenas, D., A. Artemyev, O. Agapitov, and V. Krasnoselskikh (2012a), Acceleration of radiation belts electrons by oblique chorus waves, J. Geophys. Res., 117, A10212, doi:10.1029/2012JA018041.

Mourenas, D., A. V. Artemyev, J.-F. Ripoll, O. V. Agapitov, and V. V. Krasnoselskikh (2012b), Timescales for electron quasi-linear diffusion by parallel and oblique lower-band Chorus waves, J. Geophys. Res., 117, A06234, doi:10.1029/2012JA017717.

Mourenas, D., A. V. Artemyev, O. V. Agapitov, and V. Krasnoselskikh (2013), Analytical estimates of electron quasi-linear diffusion by fast magnetosonic waves, J. Geophys. Res. Space Physics, 118, 3096-3112, doi:10.1002/jgra.50349.

Ni, B., Y. Y. Shprits, R. H. W. Friedel, R. M. Thorne, M. Daae, and Y. Chen (2013), Responses of Earth's radiation belts to solar wind dynamic pressure variations in 2002 analyzed using multi-satellite data and Kalman filtering, J. Geophys. Res. Space Physics, 118, 4400-4414, doi:10.1002/jgra.50437. 
Nunn, D., Y. Omura, H. Matsumoto, I. Nagano, and S. Yagitani (1997), The numerical simulation of VLF chorus and discrete emissions observed on the Geotail satellite using a Vlasov code, J. Geophys. Res., 102, 27,083-27,097.

O'Brien, T. P., and M. B. Moldwin (2003), Empirical plasmapause models from magnetic indices, Geophys. Res. Lett., 30(4), 1152, doi:10.1029/2002GL016007.

Omura, Y., Y. Katoh, and D. Summers (2008), Theory and simulation of the generation of whistler-mode chorus, J. Geophys. Res., 113, A04223, doi:10.1029/2007JA012622.

Reeves, G. D., K. L. McAdams, R. H. W. Friedel, and T. P. O'Brien (2003), Acceleration and loss of relativistic electrons during geomagnetic storms, Geophys. Res. Lett., 30(10), 1529, doi:10.1029/2002GL016513.

Roederer, J. G. (1970), Dynamics of Geomagnetically Trapped Radiation, Springer, New York, doi:10.1007/978-3-642-49300-3.

Santolík, O., D. A. Gurnett, J. S. Pickett, M. Parrot, and N. Cornilleau-Wehrlin (2003), Spatio-temporal structure of storm-time chorus, J. Geophys. Res., 108(A7), 1278, doi:10.1029/2002JA009791.

Santolík, O., D. A. Gurnett, J. S. Pickett, J. Chum, and N. Cornilleau-Wehrlin (2009), Oblique propagation of whistler mode waves in the chorus source region, J. Geophys. Res., 114, A00F03, doi:10.1029/2009JA014586.

Sauer, K., and R. D. Sydora (2010), Beam-excited whistler waves at oblique propagation with relation to STEREO radiation belt observations, Ann. Geophys., 28, 1317-1325, doi:10.5194/angeo-28-1317-2010.

Shapiro, V. D., and R. Z. Sagdeev (1997), Nonlinear wave-particle interaction and conditions for the applicability of quasilinear theory, Phys. Rep., 283, 49-71, doi:10.1016/S0370-1573(96)00053-1.

Sheeley, B. W., M. B. Moldwin, H. K. Rassoul, and R. R. Anderson (2001), An empirical plasmasphere and trough density model: CRRES observations, J. Geophys. Res., 106, 25,631-25,642, doi:10.1029/2000JA000286.

Shklyar, D. R. (1981), Stochastic motion of relativistic particles in the field of a monochromatic wave, Sov. Phys. JETP, 53, $1197-1192$.

Shklyar, D., and H. Matsumoto (2009), Oblique whistler-mode waves in the inhomogeneous magnetospheric plasma: Resonant interactions with energetic charged particles, Surv. Geophys., 30, 55-104, doi:10.1007/s10712-009-9061-7.

Shprits, Y. Y., R. M. Thorne, R. B. Horne, S. A. Glauert, M. Cartwright, C. T. Russell, D. N. Baker, and S. G. Kanekal (2006), Acceleration mechanism responsible for the formation of the new radiation belt during the 2003 Halloween solar storm, Geophys. Res. Lett., 33, L05104, doi:10.1029/2005GL024256.

Shprits, Y. Y., D. A. Subbotin, N. P. Meredith, and S. R. Elkington (2008), Review of modeling of losses and sources of relativistic electrons in the outer radiation belt II: Local acceleration and loss, J. Atmos. Sol. Terr. Phys., 70, 1694-1713, doi:10.1016/j.jastp.2008.06.014.

Shprits, Y. Y. (2009), Potential waves for pitch-angle scattering of near-equatorially mirroring energetic electrons due to the violation of the second adiabatic invariant, Geophys. Res. Lett., 36, L12106, doi:10.1029/2009GL038322.

Shprits, Y. Y., M. Daae, and B. Ni (2012), Statistical analysis of phase space density buildups and dropouts, J. Geophys. Res., 117, A01219, doi:10.1029/2011JA016939.

Shprits, Y. Y., D. Subbotin, A. Drozdov, M. E. Usanova, A. Kellerman, K. Orlova, D. N. Baker, D. L. Turner, and K.-C. Kim (2013), Unusual stable trapping of the ultrarelativistic electrons in the Van Allen radiation belts, Nat. Phys., 9, 699-703, doi:10.1038/nphys2760.

Spasojevic, M., and Y. Y. Shprits (2013), Chorus functional dependencies derived from CRRES data, Geophys. Res. Lett., 40, 3793-3797, doi:10.1002/grl.50755.

Summers, D., R. M. Thorne, and F. Xiao (1998), Relativistic theory of wave-particle resonant diffusion with application to electron acceleration in the magnetosphere, J. Geophys. Res., 103, 20,487-20,500, doi:10.1029/98JA01740.

Summers, D., R. Tang, and R. M. Thorne (2009), Limit on stably trapped particle fluxes in planetary magnetospheres, J. Geophys. Res., 114, A10210, doi:10.1029/2009JA014428.

Summers, D., R. Tang, and Y. Omura (2011), Effects of nonlinear wave growth on extreme radiation belt electron fluxes, J. Geophys. Res., 116, A10226, doi:10.1029/2011JA016602.

Tao, X., J. Bortnik, J. M. Albert, K. Liu, and R. M. Thorne (2011), Comparison of quasilinear diffusion coefficients for parallel propagating whistler mode waves with test particle simulations, Geophys. Res. Lett., 38, L06105, doi:10.1029/2011GL046787.

Tao, X., J. Bortnik, J. M. Albert, R. M. Thorne, and W. Li (2013), The importance of amplitude modulation in nonlinear interactions between electrons and large amplitude whistler waves, J. Atmos. Sol. Terr. Phys., 99, 67-72, doi:10.1016/j.jastp.2012.05.012.

Thorne, R. M., and C. F. Kennel (1967), Quasi-trapped VLF propagation in the outer magnetosphere, J. Geophys. Res., 72, 857-870, doi:10.1029/JZ072i003p00857.

Thorne, R. M. (2010), Radiation belt dynamics: The importance of wave-particle interactions, Geophys. Res. Lett., 372, L22107, doi:10.1029/2010GL044990.

Trakhtengerts, V. Y. (1966), Stationary states of the Earth's outer radiation zone, Geomagn. Aeron., 6, 827-836.

Tsurutani, B. T., B. J. Falkowski, O. P. Verkhoglyadova, J. S. Pickett, O. Santolík, and G. S. Lakhina (2011), Quasi-coherent chorus properties: 1. Implications for wave-particle interactions, J. Geophys. Res., 116, A09210, doi:10.1029/2010JA016237.

Tsurutani, B. T., G. S. Lakhina, and O. P. Verkhoglyadova (2013), Energetic electron (>10 keV) microburst precipitation, $\sim 5-15 \mathrm{~s}$ X-ray pulsations, chorus, and wave-particle interactions: A review, J. Geophys. Res. Space Physics, 118, 2296-2312, doi:10.1002/jgra.50264.

Turner, D. L., V. Angelopoulos, W. Li, M. D. Hartinger, M. Usanova, I. R. Mann, J. Bortnik, and Y. Shprits (2013), On the storm-time evolution of relativistic electron phase space density in Earth's outer radiation belt, J. Geophys. Res. Space Physics, 118, 2196-2212, doi:10.1002/jgra.50151.

Vette, J. I. (1991), The AE-8 trapped electron model environment, NASA STI/Recon Tech. Rep. N, 92, 24,228.

Woodroffe, J. R., and A. V. Streltsov (2013), Whistler interactions with density gradients in the magnetosphere, J. Geophys. Res. Space Physics, 118, 167-172, doi:10.1029/2012JA018308.

Yamaguchi, K., T. Matsumoto, Y. Omura, and D. Nunn (2013), Ray tracing of whistler-mode chorus elements: Implications for generation mechanisms of rising and falling tone emissions, Ann. Geophys., 31, 665-673, doi:10.5194/angeo-31-665-2013.

Yermolaev, Yu. I., and M. Yu. Yermolaev (2003), Statistical relationships between solar, interplanetary, and geomagnetic disturbances, 1976-2000, Cosmic Res., 41(6), 539-630.

Yuan, C. J., and Q.-G. Zong (2013), The double-belt outer radiation belt during CME- and CIR-driven geomagnetic storms, J. Geophys. Res. Space Physics, 118, 6291-6301, doi:10.1002/jgra.50564.

Zhao, H., and X. Li (2013), Inward shift of outer radiation belt electrons as a function of Dst index and the influence of the solar wind on electron injections into the slot region, J. Geophys. Res. Space Physics, 118, 756-764, doi:10.1029/2012JA018179. 